\title{
SUPERCONVERGENCE AND EXTRAPOLATION FOR MIXED FINITE ELEMENT METHODS ON RECTANGULAR DOMAINS
}

\author{
JUNPING WANG
}

\begin{abstract}
Asymptotic expansions for the RT (Raviart-Thomas) mixed finite element approximation by the lowest-order rectangular element associated with a second-order elliptic equation on a rectangular domain are derived. Superconvergence for the vector field along the Gauss lines is obtained as a result of the expansion. A procedure of postprocessed extrapolation is presented for the scalar field, as well as procedures of pure Richardson extrapolation for both the vector and the scalar fields.
\end{abstract}

\section{INTRODUCTION}

The aim of this paper is to discuss the asymptotic behavior of the mixed finite element approximation associated with a second-order elliptic equation. We take as our model the Dirichlet boundary value problem

$$
\begin{aligned}
-\operatorname{div}(\alpha(\mathbf{x}) \nabla u(\mathbf{x})) & =f(\mathbf{x}) & & \text { in } \Omega, \\
u & =-g(\mathbf{x}) & & \text { on } \partial \Omega,
\end{aligned}
$$

where $\Omega$ is an open bounded domain in $\mathbf{R}^{2}$ and $\partial \Omega$ the boundary of $\Omega . \alpha(\mathbf{x})$ is a positive, continuous function on $\bar{\Omega}=\Omega \cup \partial \Omega$. For the sake of simplicity of analysis, we shall take the domain $\Omega$ to be a rectangle in this paper. The results can be extended easily to Neumann boundary value problems.

A mixed form of the problem (1.1) seeks $(\mathbf{q}, u)$ such that

$$
\begin{aligned}
\beta \mathbf{q}+\nabla u & =0 & & \text { in } \Omega, \\
\operatorname{div} \mathbf{q} & =f & & \text { in } \Omega, \\
u & =-g & & \text { on } \partial \Omega,
\end{aligned}
$$

where $\beta=\beta(\mathbf{x})=\alpha^{-1}(\mathbf{x})$ is the reciprocal of the coefficient $\alpha(\mathbf{x})$.

Let $\mathbf{H}$ (div) be the Hilbert space

$$
\mathbf{H}(\operatorname{div})=\left\{\mathbf{v}: \mathbf{v} \in \mathbf{L}^{2}(\Omega), \operatorname{div} \mathbf{v} \in L^{2}(\Omega)\right\}
$$

with norm defined by

$$
\|\mathbf{v}\|_{\mathbf{H}}^{2}=\|\mathbf{v}\|_{0,2}^{2}+\|\operatorname{div} \mathbf{v}\|_{0,2}^{2},
$$

Received April 24, 1989; revised October 20, 1989 and July 12, 1990.

1980 Mathematics Subject Classification (1985 Revision). Primary 65N30, 65N15.

Key words and phrases. Second-order elliptic equation, finite element method, asymptotic expansion, superconvergence, Richardson extrapolation. 
where $\mathbf{L}^{2}(\Omega)=L^{2}(\Omega) \times L^{2}(\Omega)$ and $\|\cdot\|_{0,2}$ indicates the $L^{2}$-norm of either a scalar- or a vector-valued function. Denote by $W^{m, p}(\Omega)$ the usual Sobolev space containing functions whose partial derivatives are $L^{p}$-integrable up to order $m$. Let $\|\phi\|_{m, p}$ indicate the norm in $W^{m, p}(\Omega)$ for any function $\phi(\mathbf{x}) \epsilon$ $W^{m, p}(\Omega)$. Then a weak form of the problem (1.2) seeks $(\mathbf{q}, u) \in \mathbf{H}(\operatorname{div}) \times$ $L^{2}(\Omega)$ such that

$$
\begin{aligned}
(\beta \mathbf{q}, \mathbf{v})-(\operatorname{div} \mathbf{v}, u) & =\langle g, \mathbf{v} \cdot \mathbf{n}\rangle, & & \mathbf{v} \in \mathbf{H}(\operatorname{div}), \\
(\operatorname{div} \mathbf{q}, w) & =(f, w), & & w \in L^{2}(\Omega),
\end{aligned}
$$

where $(\cdot, \cdot)$ denotes the inner product in $L^{2}(\Omega)$ or $\mathbf{L}^{2}(\Omega)$ and $\langle\cdot, \cdot\rangle$ that in $L^{2}(\partial \Omega)$. $\mathbf{n}$ denotes the outward normal direction of $\partial \Omega$. In what follows, the two components $\mathbf{q}$ and $u$ of the solution $(\mathbf{q}, u)$ are referred to as the vector and the scalar field, respectively.

A mixed finite element method for (1.1) is based on the formula (1.3) and on finding a pair of finite element spaces $\mathbf{V}^{h} \times M^{h} \subset \mathbf{H}($ div $) \times L^{2}(\Omega)$ associated with a polygonalization $\Re_{h}$ of $\Omega$ which satisfy the inf-sup stability condition of Brezzi and Babuška (cf. $[4,1])$. In this paper, we shall consider rectangular partitions for $\Omega$ instead of a general polygonalization. Some spaces have been constructed and analyzed for rectangular elements. Among these, we would like to mention those of Raviart and Thomas [22], Brezzi, Douglas, and Marini [6], and Brezzi, Douglas, Fortin, and Marini [5]; they are referred to as RT, BDM, and BDFM spaces, respectively.

Some superconvergence results for the RT method on rectangular domains have been derived by Nakata, Weiser, and Wheeler [21] in which a discrete $L^{2}$ norm of the error arising from the Gauss points on each element is considered. One of the main objects of this paper is to discuss superconvergence of the approximation along the Gauss lines for the vector field $\mathbf{q}$ associated with the lowest-order RT method. Extensions to RT or BDFM methods of any order are given in forthcoming papers (cf. [11, 9]).

Our second aim is to discuss the asymptotic behavior of the approximation associated with the RT method of lowest order. We intend to establish an asymptotic expansion for this method. The idea of deriving an asymptotic expansion for finite element methods is not new. The earliest work, to the author's knowledge, is the one presented in Marchuk's book [20], where an expansion for the Galerkin approximation of the one-dimensional Laplace equation is derived. However, it is not clear whether the idea in [20] can be generalized to the Laplace equation in higher dimensions. Recently, Lin and $\mathrm{Lu}$ [15] presented an expansion for the Galerkin approximation for the two-dimensional Laplace equation under the assumption of uniform triangulation. The crucial idea in [15] is the use of the discrete Green's function in the representation of the Galerkin approximation. Many results have been obtained following this idea, but few of them made any essential improvement on relaxing the uniformity assumption for the triangulation. For more detailed discussions we refer to [16, $15,17,18,19,2$, etc.] and references cited therein. 
An analysis of Richardson extrapolation has been given for the mixed finite element approximation in [23]. It was proved that one can extrapolate the scalar field for the mixed finite element approximation without using any uniformity on the triangulation. Continuing [23], we shall further disscuss the extrapolation for the RT approximation in this paper. We shall illustrate that the uniformity condition is still required in extrapolating the vector field. The estimates established for the discrete Green's functions in [23] will play an important role in the forthcoming analysis. We derive asymptotic expansions and also present and analyze some procedures of extrapolation and postprocessed extrapolation; they all yield approximations of higher order to the original problem.

The idea behind the postprocessed extrapolation in this paper is the same as the postprocessing procedure presented in [23] for the lowest-order BDM method. A general local postprocessing technique (cf. [3]) was proposed by Bramble and $\mathrm{Xu}$ independent of [23]. The idea behind these procedures is the same; one tries to use the approximation of the vector field, which is proportional to the gradient of the scalar field, to get a better approximation of the scalar field via Taylor expansion.

The paper is organized as follows. In $\S 2$ we study the asymptotic behavior of a projection operator $\Pi_{h}$ and then establish a general asymptotic expansion. In $\S 3$ we present a superconvergence result as the first application of the expansion. A procedure of postprocessed extrapolation for the scalar field is presented and analyzed in $\S 4$. Finally, in $\S 5$ we present and analyze procedures of pure extrapolation for both the vector and the scalar fields.

\section{Preliminaries AND ASYMPtotic eXPANSIONS}

We shall assume throughout this paper that the domain is $\Omega=(0,1) \times(0,1)$. Let $\Delta_{x}$ and $\Delta_{y}$ be partitions of $[0,1]$,

$$
\Delta_{x}: \quad 0=x_{0}^{*}<x_{1}^{*}<x_{2}^{*}<\cdots<x_{N}^{*}=1,
$$

and

$$
\Delta_{y}: \quad 0=y_{0}^{*}<y_{1}^{*}<y_{2}^{*}<\cdots<y_{M}^{*}=1 .
$$

Denote by $I_{x}^{i}$ and $I_{y}^{j}$ the subintervals $\left(x_{i-1}^{*}, x_{i}^{*}\right)$ and $\left(y_{j-1}^{*}, y_{j}^{*}\right)$, respectively. Let $h_{i}$ or $t_{j}$ be the half-lengths of $I_{x}^{i}$ and $I_{y}^{j}$, and let

$$
h=\max _{i, j}\left\{2 h_{i}, 2 t_{j}\right\} \text {. }
$$

Set

$$
D_{y}^{j}=[0,1] \times I_{y}^{j} \text { and } D_{x}^{i}=I_{x}^{i} \times[0,1] .
$$

Let $\Re_{h}=\Delta_{x} \times \Delta_{y}=\{R\}$ be a finite element partition for the domain $\Omega$, where the element $R$ is a rectangle of the form $I_{x}^{i} \times I_{y}^{j}$ for some $I_{x}^{i} \in \Delta_{x}$ and $I_{y}^{j} \in \Delta_{y}$. A shape regularity is required for $\Re_{h}$; i.e., the ratio of the side lengths of $R$ is assumed to be bounded by a constant independent of $R$ and $h$. 


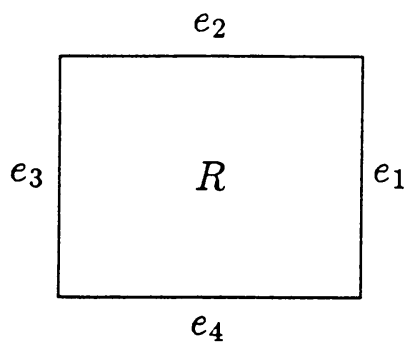

Figure 1

Illustration of rectangular element in $\mathfrak{T}_{h}$

Let us recall the definition of the RT space of the lowest order:

$$
\begin{aligned}
\mathbf{V}^{h} & =\left\{\mathbf{v}: \mathbf{v} \in \mathbf{H}(\operatorname{div}),\left.\quad \mathbf{v}\right|_{I_{x}^{i} \times I_{y}^{j}} \in P_{1}\left(I_{x}^{i}\right) \times P_{1}\left(I_{y}^{j}\right)\right\}, \\
M^{h} & =\left\{w:\left.w\right|_{I_{x}^{i} \times I_{y}^{j}} \in P_{0}\left(I_{x}^{i} \times I_{y}^{j}\right)\right\},
\end{aligned}
$$

where $P_{m}(S)$ denotes polynomials of degree $m$ on the set $S$. Let $\mathbf{H}_{r}(\mathrm{div})$ be the Banach space

$$
\mathbf{H}_{r}(\operatorname{div})=\left\{\mathbf{v}: \mathbf{v} \in \mathbf{L}^{2}(\boldsymbol{\Omega}), \operatorname{div} \mathbf{v} \in L^{r}(\boldsymbol{\Omega})\right\}
$$

equipped with a natural norm

$$
\|\mathbf{v}\|_{\mathbf{H}_{r}}=\|\mathbf{v}\|_{0,2}+\|\operatorname{div} \mathbf{v}\|_{0, r},
$$

where $r$ is any number larger than 2 .

Assume $\left(x_{i}, y_{j}\right)$ to be the center of gravity of the element $R=I_{x}^{i} \times I_{y}^{j} \in \Re_{h}$. A projection operator, denoted by $\Pi_{h}$, is defined as a linear mapping from $\mathbf{H}_{r}\left(\right.$ div) to $\mathbf{V}^{h}$ for some $r>2$ such that on each element $R \in \Re_{h}$

$$
\int_{e_{i}} \Pi_{h} \boldsymbol{\psi} \cdot \mathbf{n} d s=\int_{e_{i}} \boldsymbol{\psi} \cdot \mathbf{n} d s, \quad \boldsymbol{\psi} \in \mathbf{H}_{r}(\operatorname{div})
$$

for $i=1, \ldots, 4$, where $e_{i}$ are the four edges of the rectangle $R$ and $\mathbf{n}$ the outward normal direction on the $e_{i}$ (see Figure 1).

It can be shown that this operator $\Pi_{h}$ is well defined and satisfies the following commutativity property:

$$
\operatorname{div} \cdot \Pi_{h}=P_{h}^{0} \cdot \operatorname{div}
$$

where $P_{h}^{0}$ is the local $L^{2}$-projection operator from $L^{2}(\Omega)$ to $M^{h}$ (cf. $[22,8]$ ).

For any $\boldsymbol{\psi} \in \mathbf{H}_{r}(\mathrm{div})$, let $\Pi_{h} \boldsymbol{\psi}$ be represented on $R=I_{x}^{i} \times I_{y}^{j}$ by

$$
\Pi_{h} \boldsymbol{\psi}=\left(a_{1}+a_{2}\left(x-x_{i}\right), b_{1}+b_{2}\left(y-y_{j}\right)\right) .
$$

The coefficients $a_{i}$ and $b_{i}, i=1,2$, can be found through the relation (2.1). 
Lemma 2.1. Let $\boldsymbol{\psi} \in\left[W^{1,1}(\Omega)\right]^{2} \cap \mathbf{H}_{r}($ div $)$. Then the coefficients in the projection $\Pi_{h} \boldsymbol{\psi}$, as defined by (2.3), are given by

$$
\begin{aligned}
& a_{1}=P_{h}^{0}\left(\psi_{1}+\left(\xi-x_{i}\right) \partial_{\xi} \psi_{1}\right), \\
& a_{2}=P_{h}^{0}\left(\partial_{\xi} \psi_{1}\right), \\
& b_{1}=P_{h}^{0}\left(\psi_{2}+\left(\eta-y_{j}\right) \partial_{\eta} \psi_{2}\right), \\
& b_{2}=P_{h}^{0}\left(\partial_{\eta} \psi_{2}\right) .
\end{aligned}
$$

Proof. It suffices to check that (2.4)-(2.7) solve (2.1). Substituting (2.4), (2.5), (2.6), and (2.7) into (2.3) gives

$$
\begin{array}{r}
\Pi_{h} \boldsymbol{\psi}=\left(P_{h}^{0}\left(\psi_{1}+\left(\xi-x_{i}\right) \partial_{\xi} \psi_{1}\right)+P_{h}^{0}\left(\partial_{\xi} \psi_{1}\right)\left(x-x_{i}\right),\right. \\
\left.P_{h}^{0}\left(\psi_{2}+\left(\eta-y_{j}\right) \partial_{\eta} \psi_{2}\right)+P_{h}^{0}\left(\partial_{\eta} \psi_{2}\right)\left(y-y_{j}\right)\right) .
\end{array}
$$

We want to verify (2.1) for $\Pi_{h} \psi$ given by (2.8). It follows from (2.8) that

$$
\begin{aligned}
\int_{e_{1}} \Pi_{h} \boldsymbol{\psi} \cdot \mathbf{n} d s & =\int_{e_{1}}\left[P_{h}^{0}\left(\psi_{1}+\left(\xi-x_{i}\right) \partial_{\xi} \psi_{1}\right)+P_{h}^{0}\left(\partial_{\xi} \psi_{1}\right)\left(x-x_{i}\right)\right] d s \\
& =\left|e_{1}\right|\left\{P_{h}^{0}\left(\psi_{1}+\left(\xi-x_{i}\right) \partial_{\xi} \psi_{1}\right)+h_{i} P_{h}^{0}\left(\partial_{\xi} \psi_{1}\right)\right\} \\
& =\left|e_{1}\right| P_{h}^{0}\left\{\partial_{\xi}\left[\left(\xi-x_{i}\right) \psi_{1}\right]+h_{i} \partial_{\xi} \psi_{1}\right\} \\
& =\left|e_{1}\right| P_{h}^{0}\left\{\partial_{\xi}\left[\left(\left(\xi-x_{i}\right)+h_{i}\right) \psi_{1}\right]\right\} \\
& =\frac{\left|e_{1}\right|}{|R|} \int_{R} \partial_{\xi}\left[\left(\left(\xi-x_{i}\right)+h_{i}\right) \psi_{1}\right] d \xi d \eta \\
& =\frac{2 h_{i}\left|e_{1}\right|}{|R|} \int_{e_{1}} \psi_{1} d s=\int_{e_{1}} \psi_{1} d s,
\end{aligned}
$$

which implies that

$$
\int_{e_{1}} \Pi_{h} \boldsymbol{\psi} \cdot \mathbf{n} d s=\int_{e_{1}} \boldsymbol{\psi} \cdot \mathbf{n} d s .
$$

The same argument can be applied for the other three edges to verify the conclusion of the lemma.

It follows from Lemma 2.1 that $\Pi_{h} \boldsymbol{\psi}$ is defined componentwise. Thus, we can define the projection of each component of any vector-valued function and shall denote by $\Pi_{h} \psi_{i}$ the $i$ th component of the projection of $\boldsymbol{\psi}$.

We are now in a position to derive an asymptotic expansion for the error of the mixed finite element approximation of (1.3) using an RT space of the lowest order.

Lemma 2.2. Let $\mathbf{q} \in\left[P_{2}(R)\right]^{2}$ with $R=I_{x}^{i} \times I_{y}^{j} \in \Re_{h}$. Then,

$$
\Pi_{h} q_{1}(x, y)=q_{1}\left(x, y_{j}\right)-\frac{1}{2}\left(x-x_{i}\right)^{2} \partial_{x}^{2} q_{1}+\frac{h_{i}^{2}}{2} \partial_{x}^{2} q_{1}+\frac{t_{j}^{2}}{6} \partial_{y}^{2} q_{1}
$$


and

$$
\Pi_{h} q_{2}(x, y)=q_{2}\left(x_{i}, y\right)-\frac{1}{2}\left(y-y_{j}\right)^{2} \partial_{y}^{2} q_{2}+\frac{t_{j}^{2}}{2} \partial_{y}^{2} q_{2}+\frac{h_{i}^{2}}{6} \partial_{x}^{2} q_{2} .
$$

Proof. By Lemma 2.1,

$$
\Pi_{h} q_{1}=P_{h}^{0}\left(q_{1}+\left(\xi-x_{i}\right) \partial_{\xi} q_{1}\right)+P_{h}^{0}\left(\partial_{\xi} q_{1}\right)\left(x-x_{i}\right) \text { on } R \text {. }
$$

Since

$$
\begin{aligned}
P_{h}^{0}\left(\left(\xi-x_{i}\right) \partial_{\xi} q_{1}\right) & =\frac{1}{|R|} \int_{R}\left(\xi-x_{i}\right) \partial_{\xi} q_{1} d \xi d \eta \\
& =\frac{1}{|R|} \int_{R}\left(\xi-x_{i}\right)^{2} \partial_{\xi}^{2} q_{1} d \xi d \eta=\frac{h_{i}^{2}}{3} \partial_{x}^{2} q_{1}
\end{aligned}
$$

we have

$$
\Pi_{h} q_{1}=P_{h}^{0} q_{1}+\left(x-x_{i}\right) P_{h}^{0}\left(\partial_{\xi} q_{1}\right)+\frac{h_{i}^{2}}{3} \partial_{x}^{2} q_{1} .
$$

Further, by a Taylor expansion,

$$
\begin{aligned}
P_{h}^{0} q_{1}= & \frac{1}{|R|} \int_{R} q_{1}(\xi, \eta) d \xi d \eta \\
= & q_{1}\left(x, y_{j}\right)+\frac{1}{|R|} \int_{R}(\xi-x) d \xi d \eta \partial_{x} q_{1}\left(x, y_{j}\right) \\
& +\frac{1}{2|R|}\left(\int_{R}(\xi-x)^{2} d \xi d \eta \partial_{x}^{2} q_{1}+\int_{R}\left(\eta-y_{j}\right)^{2} d \xi d \eta \partial_{y}^{2} q_{1}\right) \\
= & q_{1}\left(x, y_{j}\right)+\left(x_{i}-x\right) \partial_{x} q_{1}\left(x, y_{j}\right) \\
& +\left(\frac{1}{2}\left(x-x_{i}\right)^{2}+\frac{h_{i}^{2}}{6}\right) \partial_{x}^{2} q_{1}+\frac{t_{j}^{2}}{6} \partial_{y}^{2} q_{1} .
\end{aligned}
$$

Thus, substituting (2.14) into (2.13) gives

$$
\begin{aligned}
\Pi_{h} q_{1}= & q_{1}\left(x, y_{j}\right)+\left(x_{i}-x\right) \partial_{x} q_{1}\left(x, y_{j}\right)+\frac{1}{2}\left(x-x_{i}\right)^{2} \partial_{x}^{2} q_{1}+\frac{h_{i}^{2}}{6} \partial_{x}^{2} q_{1} \\
& +\frac{t_{j}^{2}}{6} \partial_{y}^{2} q_{1}+\left(x-x_{i}\right) P_{h}^{0}\left(\partial_{\xi} q_{1}\right)+\frac{h_{i}^{2}}{3} \partial_{x}^{2} q_{1} \\
= & q_{1}\left(x, y_{j}\right)+\left(x_{i}-x\right) \partial_{x} q_{1}\left(x, y_{j}\right)+\frac{1}{2}\left(x-x_{i}\right)^{2} \partial_{x}^{2} q_{1}+\frac{h_{i}^{2}}{2} \partial_{x}^{2} q_{1} \\
& +\frac{t_{j}^{2}}{6} \partial_{y}^{2} q_{1}+\left(x-x_{i}\right) \partial_{x} q_{1}\left(x_{i}, y_{j}\right) \\
= & q_{1}\left(x, y_{j}\right)-\left(x-x_{i}\right)^{2} \partial_{x}^{2} q_{1}+\frac{1}{2}\left(x-x_{i}\right)^{2} \partial_{x}^{2} q_{1}+\frac{h_{i}^{2}}{2} \partial_{x}^{2} q_{1}+\frac{t_{j}^{2}}{6} \partial_{y}^{2} q_{1} \\
= & q_{1}\left(x, y_{j}\right)-\frac{1}{2}\left(x-x_{i}\right)^{2} \partial_{x}^{2} q_{1}+\frac{h_{i}^{2}}{2} \partial_{x}^{2} q_{1}+\frac{t_{j}^{2}}{6} \partial_{y}^{2} q_{1},
\end{aligned}
$$

so that (2.11) holds. The same arguments can be applied to verify (2.12). 
Theorem 2.1. Let $\mathbf{q} \in \mathbf{H}_{r}(\mathrm{div})$. Let $\Re_{h}$ be the partition of $\Omega$ given at the beginning of this section. Let $\beta \in W^{2, \infty}(\Omega)$. Then, for any $\mathbf{v}=\left(v_{1}, v_{2}\right) \in \mathbf{V}^{h}$,

$$
\begin{aligned}
\left(\beta\left(\mathbf{q}-\Pi_{h} \mathbf{q}\right), \mathbf{v}\right)= & \frac{1}{3} \sum_{i=1}^{N} h_{i}^{2} \int_{D_{x}^{i}}\left(\partial_{x} \beta \partial_{x} q_{2} v_{2}-\beta \partial_{x}^{2} q_{1} v_{1}\right) d x d y \\
& +\frac{1}{3} \sum_{j=1}^{M} t_{j}^{2} \int_{D_{y}^{j}}\left(\partial_{y} \beta \partial_{y} q_{1} v_{1}-\beta \partial_{y}^{2} q_{2} v_{2}\right) d x d y+R(\mathbf{v}),
\end{aligned}
$$

where

$$
|R(\mathbf{v})| \leq C h^{3}\|\mathbf{q}\|_{3, p}\|\mathbf{v}\|_{p^{\prime}},
$$

provided that $\mathbf{q} \in\left[W^{3, p}\right]^{2}$ with $p \geq 1$ and $1 / p+1 / p^{\prime}=1$.

Proof. Since

$$
\left(\beta\left(\mathbf{q}-\Pi_{h} \mathbf{q}\right), \mathbf{v}\right)=\sum_{i, j} \int_{I_{x}^{i} \times I_{y}^{j}} \beta\left(\mathbf{q}-\Pi_{h} \mathbf{q}\right) \cdot \mathbf{v} d x d y,
$$

it is sufficient to analyze

$$
\int_{I_{x}^{i} \times I_{y}^{j}} \beta\left(\mathbf{q}-\Pi_{h} \mathbf{q}\right) \cdot \mathbf{v} d x d y=\int_{R} \beta\left(\mathbf{q}-\Pi_{h} \mathbf{q}\right) \cdot \mathbf{v} d x d y=U_{1}+U_{2},
$$

where

$$
\begin{aligned}
& U_{1}=\int_{R} \beta\left(q_{1}-\Pi_{h} q_{1}\right) v_{1} d x d y, \\
& U_{2}=\int_{R} \beta\left(q_{2}-\Pi_{h} q_{2}\right) v_{2} d x d y .
\end{aligned}
$$

We may assume, without loss of generality, that $q_{i}(i=1,2)$ are polynomials of degree not exceeding two on each $R$. Then, by (2.11),

$$
\begin{aligned}
U_{1}= & \int_{R} \beta\left(q_{1}(x, y)-q_{1}\left(x, y_{j}\right)\right) v_{1} d x d y \\
& +\frac{1}{2} \int_{R} \beta\left(x-x_{i}\right)^{2} \partial_{x}^{2} q_{1} v_{1} d x d y \\
& \quad-\frac{h_{i}^{2}}{2} \int_{R} \beta \partial_{x}^{2} q_{1} v_{1} d x d y-\frac{t_{j}^{2}}{6} \int_{R} \beta \partial_{y}^{2} q_{1} v_{1} d x d y \\
= & I_{1}+I_{2}-I_{3}-I_{4} .
\end{aligned}
$$

Set

$$
\begin{aligned}
R_{6,1}^{i j}\left(v_{1}\right)= & \int_{R}\left(\beta^{I}-\beta\right)\left(\frac{t_{j}^{2}}{6} \partial_{y}^{2} q_{1}+q_{1}\left(x, y_{j}\right)-q_{1}(x, y)\right) v_{1} d x d y \\
& +\frac{t_{j}^{2}}{3} \int_{R} \partial_{y}\left(\beta^{I}-\beta\right) \partial_{y} q_{1} v_{1} d x d y,
\end{aligned}
$$




$$
R_{6,2}^{i j}\left(v_{1}\right)=\frac{1}{2} \int_{R}(\beta-\bar{\beta})\left(\left(x-x_{i}\right)^{2}-\frac{h_{i}^{2}}{3}\right) \partial_{x}^{2} q_{1} v_{1} d x d y,
$$

and

$$
v_{1}=c_{0}+c_{1}\left(x-x_{i}\right),
$$

where $\bar{\beta}$ and $\beta^{I}$ denote the average and the bilinear interpolant of $\beta$ on $R$, respectively. Note that

$$
\begin{aligned}
I_{1}= & \int_{R} \beta^{I}\left(q_{1}(x, y)-q_{1}\left(x, y_{j}\right)\right) v_{1} d x d y \\
& +\int_{R}\left(\beta-\beta^{I}\right)\left(q_{1}(x, y)-q_{1}\left(x, y_{j}\right)\right) v_{1} d x d y .
\end{aligned}
$$

Since for any fixed $x$, the function $\beta^{I}\left(q_{1}(x, \cdot)-q_{1}\left(x, y_{j}\right)\right)$ is a polynomial of degree 3 in $y$, it is easy to see that

$$
\begin{aligned}
\int_{R} \beta^{I} & \left(q_{1}(x, y)-q_{1}\left(x, y_{j}\right)\right) v_{1} d x d y \\
& =\int_{x}\left(\int_{y_{j}-t_{j}}^{y_{j}+t_{j}} \beta^{I}\left(q_{1}(x, y)-q_{1}\left(x, y_{j}\right)\right) v_{1} d y\right) d x \\
& =\frac{t_{j}^{3}}{3} \int_{x} \partial_{y}^{2}\left(\beta^{I}\left(q_{1}(x, y)-q_{1}\left(x, y_{j}\right)\right) v_{1}\right)\left(x, y_{j}\right) d x \\
& =\frac{t_{j}^{2}}{6} \int_{R} \beta^{I} \partial_{y}^{2} q_{1} v_{1} d x d y+\frac{t_{j}^{2}}{3} \int_{R} \partial_{y} \beta^{I} \partial_{y} q_{1} v_{1} d x d y .
\end{aligned}
$$

Combining (2.24) with (2.25) and (2.22) gives

$$
\begin{aligned}
I_{1}= & \frac{t_{j}^{2}}{6} \int_{R} \beta^{I} \partial_{y}^{2} q_{1} v_{1} d x d y+\frac{t_{j}^{2}}{3} \int_{R} \partial_{y} \beta^{I} \partial_{y} q_{1} v_{1} d x d y \\
& \quad+\int_{R}\left(\beta-\beta^{I}\right)\left(q_{1}(x, y)-q_{1}\left(x, y_{j}\right)\right) v_{1} d x d y \\
= & \frac{t_{j}^{2}}{6} \int_{R} \beta \partial_{y}^{2} q_{1} v_{1} d x d y+\frac{t_{j}^{2}}{3} \int_{R} \partial_{y} \beta \partial_{y} q_{1} v_{1} d x d y+R_{6,1}^{i j}\left(v_{1}\right) .
\end{aligned}
$$

As to $I_{2}$, we observe

$$
\begin{gathered}
\int_{R} \bar{\beta}\left(x-x_{i}\right)^{2} \partial_{x}^{2} q_{1} v_{1} d x d y=\int_{R}\left(x-x_{i}\right)^{2}\left(c_{0}+c_{1}\left(x-x_{i}\right)\right) d x d y \bar{\beta} \partial_{x}^{2} q_{1} \\
=\int_{R}\left(x-x_{i}\right)^{2} c_{0} d x d y \bar{\beta} \partial_{x}^{2} q_{1}=\frac{h_{i}^{2}}{3} \int_{R} c_{0} \bar{\beta} \partial_{x}^{2} q_{1} d x d y \\
=\frac{h_{i}^{2}}{3} \int_{R} \bar{\beta} \partial_{x}^{2} q_{1}\left(c_{0}+c_{1}\left(x-x_{i}\right)\right) d x d y=\frac{h_{i}^{2}}{3} \int_{R} \bar{\beta} \partial_{x}^{2} q_{1} v_{1} d x d y
\end{gathered}
$$


which together with $(2.23)$ yields

$$
\begin{aligned}
I_{2}= & \frac{1}{2} \int_{R} \bar{\beta}\left(x-x_{i}\right)^{2} \partial_{x}^{2} q_{1} v_{1} d x d y \\
& +\frac{1}{2} \int_{R}(\beta-\bar{\beta})\left(x-x_{i}\right)^{2} \partial_{x}^{2} q_{1} v_{1} d x d y \\
= & \frac{h_{i}^{2}}{6} \int_{R} \bar{\beta} \partial_{x}^{2} q_{1} v_{1} d x d y+\frac{1}{2} \int_{R}(\beta-\bar{\beta})\left(x-x_{i}\right)^{2} \partial_{x}^{2} q_{1} v_{1} d x d y \\
= & \frac{h_{i}^{2}}{6} \int_{R} \beta \partial_{x}^{2} q_{1} v_{1} d x d y+R_{6,2}^{i j}\left(v_{1}\right) .
\end{aligned}
$$

Thus, combining (2.21) with (2.26) and (2.28) gives

$$
U_{1}=\frac{t_{j}^{2}}{3} \int_{R} \partial_{y} \beta \partial_{y} q_{1} v_{1} d x d y-\frac{h_{i}^{2}}{3} \int_{R} \beta \partial_{x}^{2} q_{1} v_{1} d x d y+R_{6,1}^{i j}\left(v_{1}\right)+R_{6,2}^{i j}\left(v_{1}\right)
$$

The same argument leads to

$$
\begin{aligned}
U_{2}= & \frac{h_{i}^{2}}{3} \int_{R} \partial_{x} \beta \partial_{x} q_{2} v_{2} d x d y \\
& -\frac{t_{j}^{2}}{3} \int_{R} \beta \partial_{y}^{2} q_{2} v_{2} d x d y+R_{7,1}^{i j}\left(v_{2}\right)+R_{7,2}^{i j}\left(v_{2}\right),
\end{aligned}
$$

where

$$
\begin{aligned}
R_{7,1}^{i j}\left(v_{2}\right)= & \int_{R}\left(\beta^{I}-\beta\right)\left(\frac{h_{i}^{2}}{6} \partial_{x}^{2} q_{2}+q_{2}\left(x_{i}, y\right)-q_{2}(x, y)\right) v_{2} d x d y \\
& +\frac{h_{i}^{2}}{3} \int_{R} \partial_{x}\left(\beta^{I}-\beta\right) \partial_{x} q_{2} v_{2} d x d y
\end{aligned}
$$

and

$$
R_{7,2}^{i j}\left(v_{2}\right)=\frac{1}{2} \int_{R}(\beta-\bar{\beta})\left(\left(y-y_{j}\right)^{2}-\frac{t_{j}^{2}}{3}\right) \partial_{y}^{2} q_{2} v_{2} d x d y
$$

Let

$$
R_{6}(\mathbf{v})=\sum_{k=1}^{2} \sum_{i, j}\left(R_{6, k}^{i j}\left(v_{1}\right)+R_{7, k}^{i j}\left(v_{2}\right)\right) .
$$

It follows from (2.17) together with (2.18), (2.29), and (2.30) that

$$
\begin{aligned}
\left(\beta\left(\mathbf{q}-\Pi_{h} \mathbf{q}\right), \mathbf{v}\right)= & \frac{1}{3} \sum_{j=1}^{M} t_{j}^{2} \int_{D_{y}^{j}}\left(\partial_{y} \beta \partial_{y} q_{1} v_{1}-\beta \partial_{y}^{2} q_{2} v_{2}\right) d x d y \\
& +\frac{1}{3} \sum_{i=1}^{N} h_{i}^{2} \int_{D_{x}^{i}}\left(\partial_{x} \beta \partial_{x} q_{2} v_{2}-\beta \partial_{x}^{2} q_{1} v_{1}\right) d x d y+R_{6}(\mathbf{v})
\end{aligned}
$$

with

$$
\left|R_{6}(\mathbf{v})\right| \leq C h^{3}\|\mathbf{q}\|_{2, p}\|\mathbf{v}\|_{p^{\prime}}
$$


Now, the conclusion of Theorem 2.1 follows from (2.34), (2.35), and the Bramble-Hilbert lemma.

Remark 2.1. Assume that $\mathbf{q} \in\left[W^{2, p}\right]^{2}$ with $p \geq 1$. From the proof of Theorem 2.1 it is not difficult to see that

$$
\left|\left(\beta\left(\mathbf{q}-\Pi_{h} \mathbf{q}\right), \mathbf{v}\right)\right| \leq C h^{2}\|\mathbf{q}\|_{2, p}\|\mathbf{v}\|_{p^{\prime}}, \quad \mathbf{v} \in \mathbf{V}^{h},
$$

where $p^{\prime}=p /(p-1)$ is the conjugate of $p$.

Two particular choices of $\mathbf{v}$ and $p$ will yield asymptotic expansions for either the scalar or the vector field of the mixed finite element approximation of (1.3). Therefore, (2.15) can be viewed as a general error expansion for lowest-order RT mixed finite element approximations.

\section{SUPERCONVERGENCE}

In this section, we apply the asymptotic error expansion (2.15) to obtain superconvergence for the approximation of the vector field along the Gauss lines, rather than only at the Gauss points, as in [21]. We begin with the following result.

Theorem 3.1. Let $\left(\mathbf{q}^{h}, u^{h}\right)$ be the mixed finite element approximation of (1.3) lying in the $R T$ space of lowest order. Assume that $\mathbf{q} \in\left[W^{2, p}(\Omega)\right]^{2}$ with $p \geq 1$. Then

$$
\left\|\mathbf{q}^{h}-\Pi_{h} \mathbf{q}\right\|_{0,2} \leq C h^{\tau}\|\mathbf{q}\|_{2, p},
$$

where $\tau=\min \left\{2,1+2 / p^{\prime}\right\}$ and $p^{\prime}=p /(p-1)$ is the conjugate of $p$.

Proof. Set $\mathbf{v}=\mathbf{q}^{h}-\Pi_{h} \mathbf{q}$ and recall that $\operatorname{div} \mathbf{v}=0$. Thus, by relation (2.2) and the inverse inequality,

$$
\begin{aligned}
\left\|\mathbf{q}^{h}-\Pi_{h} \mathbf{q}\right\|_{0,2}^{2} & \leq C(\beta \mathbf{v}, \mathbf{v})=C\left(\beta \mathbf{v}, \mathbf{q}-\Pi_{h} \mathbf{q}\right) \\
& \leq C h^{2}\|\mathbf{q}\|_{2, p}\|\mathbf{v}\|_{0, p^{\prime}} \\
& \leq C h^{2+\min (0,1-2 / p)}\|\mathbf{q}\|_{2, p}\left\|\mathbf{q}^{h}-\Pi_{h} \mathbf{q}\right\|_{0,2},
\end{aligned}
$$

where we have used (2.36) in the second line. Thus, (3.1) follows from (3.2).

As an immediate consequence of (3.1), we have the following result.

Corollary 3.1. In addition to the assumptions of Theorem 3.1, let $\mathbf{q} \in\left[W^{1, \infty}(\Omega)\right]^{2}$ for $p \geq 2$. Then

$$
\left\|\mathbf{q}-\mathbf{q}^{h}\right\|_{0, \infty} \leq \begin{cases}C(p) h^{\frac{2}{p}}\|\mathbf{q}\|_{2, p}, & 1<p<2, \\ C h\left(\|\mathbf{q}\|_{1, \infty}+\|\mathbf{q}\|_{2, p}\right), & p \geq 2 .\end{cases}
$$

Proof. Clearly,

$$
\left\|\mathbf{q}-\mathbf{q}^{h}\right\|_{0, \infty} \leq\left\|\mathbf{q}-\Pi_{h} \mathbf{q}\right\|_{0, \infty}+\left\|\Pi_{h} \mathbf{q}-\mathbf{q}^{h}\right\|_{0, \infty} .
$$


By the standard inverse inequality and (3.1),

$$
\begin{aligned}
\left\|\Pi_{h} \mathbf{q}-\mathbf{q}^{h}\right\|_{0, \infty} & \leq C h^{-1}\left\|\Pi_{h} \mathbf{q}-\mathbf{q}^{h}\right\|_{0,2} \\
& \leq C h^{-1} h^{\tau}\|\mathbf{q}\|_{2, p} \leq C h^{\tau-1}\|\mathbf{q}\|_{2, p} .
\end{aligned}
$$

Interpolation theory and the Sobolev inequality imply that

$$
\begin{aligned}
\left\|\mathbf{q}-\Pi_{h} \mathbf{q}\right\|_{0, \infty} & \leq \begin{cases}C h^{\frac{2}{p}}\|\mathbf{q}\|_{1, \frac{2 p}{2-p}}, & 1<p<2, \\
C h\|\mathbf{q}\|_{1, \infty}, & p \geq 2,\end{cases} \\
& \leq \begin{cases}C(p) h_{p}^{\frac{2}{p}}\|\mathbf{q}\|_{2, p}, & 1<p<2, \\
C h\|\mathbf{q}\|_{1, \infty}, & p \geq 2 .\end{cases}
\end{aligned}
$$

Thus, (3.3) follows from (3.4) combined with (3.5) and (3.6).

With the help of (2.36) we are able to obtain a superconvergence result in the maximum norm for the vector field of the lowest-order mixed finite element approximation. It should be noted that all these analyses make strong use of the $L^{1}$-error estimates for the regularized Green's functions (see Theorem 2 of the Appendix).

Lemma 3.1. Let $\left(\mathbf{q}^{h}, u^{h}\right)$ be the mixed finite element approximation of (1.3) in the $R T$ space of the lowest order. Assume that $\mathbf{q} \in\left[W^{2, p}(\Omega)\right]^{2}$ with $p \geq 1$. Then

$$
\left\|\mathbf{q}^{h}-\Pi_{h} \mathbf{q}\right\|_{0, \infty} \leq \begin{cases}C h^{\frac{2}{p^{2}}} \log \frac{1}{h}\|\mathbf{q}\|_{2, p}, & p>2, \\ C h^{\frac{2}{p}}\|\mathbf{q}\|_{2, p}, & 1 \leq p \leq 2,\end{cases}
$$

where $p^{\prime}=p /(p-1)$.

Proof. By (A.13),

$$
\left\|\mathbf{q}^{h}-\Pi_{h} \mathbf{q}\right\|_{0, \infty} \leq\left|\left(\beta\left(\mathbf{q}-\Pi_{h} \mathbf{q}\right), \mathbf{G}_{2}^{h}\right)\right| .
$$

Further, by (2.36),

$$
\left|\left(\beta\left(\mathbf{q}-\Pi_{h} \mathbf{q}\right), \mathbf{G}_{2}^{h}\right)\right| \leq C h^{2}\|\mathbf{q}\|_{2, p}\left\|\mathbf{G}_{2}^{h}\right\|_{0, p^{\prime}} .
$$

Thus, it follows from (A.9) that

$$
\left|\left(\beta\left(\mathbf{q}-\Pi_{h} \mathbf{q}\right), \mathbf{G}_{2}^{h}\right)\right| \leq \begin{cases}C h^{\frac{2}{p}} \log \frac{1}{h}\|\mathbf{q}\|_{2, p}, & p>2, \\ C h^{\frac{2}{p}}\|\mathbf{q}\|_{2, p}, & 1 \leq p \leq 2,\end{cases}
$$

which, along with (3.8), implies (3.7).

To see how $\mathbf{q}^{h}$ approximates $\mathbf{q}$, it suffices to observe the approximation property of $\Pi_{h}$. We already know that $\Pi_{h} \mathbf{q}$ approximates $\mathbf{q}$ globally to an order of $O(h)$. However, from (2.11) it is easy to see that the error between 
$\Pi_{h} q_{1}$ and $q_{1}$ has an order of $O\left(h^{2}\right)$ along the Gauss lines $[0,1] \times y_{j}$ on the element $I_{x}^{i} \times I_{y}^{j}$. Let

Set

$$
\begin{aligned}
& \Gamma_{11}^{h}=[0,1] \times\left\{y_{j} ; j=1,2, \ldots, M\right\}, \\
& \Gamma_{12}^{h}=[0,1] \times\left\{y_{j}^{*} ; j=1,2, \ldots, M-1\right\}, \\
& \Gamma_{21}^{h}=\left\{x_{i} ; i=1,2, \ldots, N\right\} \times[0,1], \\
& \Gamma_{22}^{h}=\left\{x_{i}^{*} ; i=1,2, \ldots, N-1\right\} \times[0,1] .
\end{aligned}
$$

For any $\mathbf{v}=\left(v_{1}, v_{2}\right) \in \mathbf{H}(\operatorname{div}), \mathbf{v}^{*}=\left(v_{1}^{*}, v_{2}^{*}\right)$ is defined on $\Gamma^{h}=\Gamma_{1}^{h} \cup \Gamma_{2}^{h}$ as follows: Define $v_{1}^{*}$ on $\Gamma_{1}^{h}$ by

$$
v_{1}^{*}(x, y)=v_{1}(x, y), \quad(x, y) \in \Gamma_{11}^{h},
$$

and

$$
v_{1}^{*}\left(x, y_{j}^{*}\right)=\frac{1}{t_{j}+t_{j+1}}\left[t_{j} v_{1}^{+}\left(x, y_{j}^{*}\right)+t_{j+1} v_{1}^{-}\left(x, y_{j}^{*}\right)\right], \quad\left(x, y_{j}^{*}\right) \in \Gamma_{12}^{h},
$$

where

$$
v_{1}^{+}\left(x, y_{j}^{*}\right)=\lim _{y \backslash y_{j}^{*}} v_{1}(x, y),
$$

and $v_{1}^{-}\left(x, y_{j}^{*}\right)$ is defined similarly; a function $v_{2}^{*}$ can be defined analogously on $\Gamma_{2}^{h}$.

Denote by $\|\boldsymbol{\psi}\|_{\infty}$ a seminorm for piecewise continuous vector-valued function $\psi$ such that

$$
\|\boldsymbol{\psi}\|_{\infty}=\sum_{i=1}^{2}\left\|\boldsymbol{\psi}_{i}\right\|_{0, \infty, \Gamma_{i}^{h}}
$$

Now we are ready to establish the superconvergence result along the Gauss lines.

Theorem 3.2. Assume the hypotheses of Lemma 3.1. Then

$$
\left\|\mathbf{q}-\left(\mathbf{q}^{h}\right)^{*}\right\|_{\infty} \leq \begin{cases}C h^{\frac{2}{7}} \log \frac{1}{h}\|\mathbf{q}\|_{2, p}, & p>2, \\ C h^{\frac{2}{p}}\|\mathbf{q}\|_{2, p}, & 1<p \leq 2 .\end{cases}
$$

Proof. By (3.7) and the definition of $\mathbf{v}^{*}$, it suffices to check that

$$
\left\|q_{i}-\left(\Pi_{h} q_{i}\right)^{*}\right\|_{0, \infty, \Gamma_{i}^{h}} \leq C h^{p^{+}}\|\mathbf{q}\|_{2, p}, \quad i=1,2 .
$$

The following result can be proved easily through an application of (2.11):

$$
\Pi_{h} q_{1}(x, y)=q_{1}\left(x, y_{j}\right)+O\left(h^{\frac{2}{p}}\right)\left\|q_{1}\right\|_{2, p} \quad \text { on } R=I_{x}^{i} \times I_{y}^{j} .
$$

Thus, it follows from (3.20) that

$$
\left\|q_{1}-\left(\Pi_{h} q_{1}\right)^{*}\right\|_{0, \infty, \Gamma_{11}^{h}} \leq C h^{\frac{2}{p}}\left\|q_{1}\right\|_{2, p},
$$

since, by (3.16), $\left(\Pi_{h} q_{1}\right)^{*}=\Pi_{h} q_{1}$ holds on $\Gamma_{11}^{h}$. 
Let $\left(x, y_{j}^{*}\right) \in \Gamma_{12}^{h}$. By (3.20) and (3.17),

$$
\begin{aligned}
\left(\Pi_{h} q_{1}\right)^{*}\left(x, y_{j}^{*}\right)= & \frac{1}{t_{j}+t_{j+1}}\left[t_{j} q_{1}\left(x, y_{j+1}\right)+t_{j+1} q_{1}\left(x, y_{j}\right)\right] \\
& +O\left(h^{\frac{2}{p}}\right)\left\|q_{1}\right\|_{2, p} \\
= & q_{1}\left(x, y_{j}^{*}\right)+O\left(h^{\frac{2}{p}}\right)\left\|q_{1}\right\|_{2, p},
\end{aligned}
$$

so that (3.19) holds on $\Gamma_{12}^{h}$, and the theorem is proved.

\section{PostProcessed extRAPOLATION FOR THE PRESSURE}

We now investigate the validity of the technique of extrapolation for the approximation of the scalar field. We first derive an asymptotic error expansion for the approximation by using the general expansion (2.15), and then define and analyze a procedure of postprocessed extrapolation.

To derive an asymptotic error expansion for the scalar field, let $\Re_{h}$ be the partition defined at the beginning of $\S 2$. Let $\left(\mathbf{q}^{h}, u^{h}\right)$ be the mixed finite element approximation of the problem (1.3) in the lowest-order RT space.

Lemma 4.1. Let $p$ be any number in $(1,2]$ and assume that $\mathbf{q} \in\left[W^{3, p}(\Omega)\right]^{2}$ for $p<2$ and $\mathbf{q} \in\left[W^{3,2}(\Omega)\right]^{2} \cap\left[W^{2, \infty}(\Omega)\right]^{2}$ for $p=2$. Then there exists $a$ function $E_{1}^{h}(\mathbf{x})$ such that

$$
u^{h}(\mathbf{x})=P_{h}^{0} u(\mathbf{x})+\sum_{i=1}^{N} h_{i}^{2} \int_{D_{x}^{i}} D_{1} \mathbf{q} \cdot \mathbf{G}_{1} d x d y+\sum_{j=1}^{M} t_{j}^{2} \int_{D_{y}^{j}} D_{2} \mathbf{q} \cdot \mathbf{G}_{1} d x d y+E_{1}^{h}(\mathbf{x})
$$

with

$$
\left\|E_{1}^{h}\right\|_{0, \infty} \leq \begin{cases}C h^{3} \log \frac{1}{h}\left(\|\mathbf{q}\|_{2, \infty}+\|\mathbf{q}\|_{3,2}\right), & p=2, \\ C(p) h^{2+\frac{2}{p}} \log \frac{1}{h}\|\mathbf{q}\|_{3, p}, & 1<p<2\end{cases}
$$

where

$$
D_{1} \mathbf{q}=\frac{1}{3}\left(\beta \partial_{x}^{2} q_{1},-\partial_{x} \beta \partial_{x} q_{2}\right)
$$

and

$$
D_{2} \mathbf{q}=\frac{1}{3}\left(\beta \partial_{y}^{2} q_{2},-\partial_{y} \beta \partial_{y} q_{1}\right)
$$

are two vector-valued functions. $\left(\mathbf{G}_{1}, \lambda_{1}\right)$ is the regularized Green's function of the first kind in mixed form.

Proof. Since both $u^{h}$ and $P_{h}^{0} u$ are constants on each element $R \in \Re_{h}$, we need only establish (4.1) for $\mathbf{x}=(x, y)$ being the centers of the elements in $\Re_{h}$. Let $\left(x_{i}, y_{j}\right)$ be the center of gravity of the element $I_{x}^{i} \times I_{y}^{j}$. It follows from (A.1) that

$$
u^{h}\left(x_{i}, y_{j}\right)-P_{h}^{0} u\left(x_{i}, y_{j}\right)=\left(u^{h}-P_{h}^{0} u, \delta_{1}^{h}\right)
$$


where $\delta_{1}^{h}$ is the regularized Dirac $\delta$-function associated with the space $M^{h}$ and the point $\left(x_{i}, y_{j}\right)$. By the identity (A.10),

$$
u^{h}\left(x_{i}, y_{j}\right)=P_{h}^{0} u\left(x_{i}, y_{j}\right)+\left(\beta\left(\Pi_{h} \mathbf{q}-\mathbf{q}\right), \mathbf{G}_{1}^{h}\right),
$$

where $\mathbf{G}_{1}^{h}$ is the mixed finite element approximation of $\mathbf{G}_{1}$ (see the Appendix for its definition). Thus, an application of (2.15), with $\mathbf{v}$ replaced by $\mathbf{G}_{1}^{h}$, leads to

$$
\begin{aligned}
u^{h}\left(x_{i}, y_{j}\right)= & P_{h}^{0} u\left(x_{i}, y_{j}\right) \\
& +\frac{1}{3} \sum_{i=1}^{N} h_{i}^{2} \int_{D_{x}^{i}}\left(\beta \partial_{x}^{2} q_{1}\left(\mathbf{G}_{1}^{h}\right)_{1}-\partial_{x} \beta \partial_{x} q_{2}\left(\mathbf{G}_{1}^{h}\right)_{2}\right) d x d y \\
& +\frac{1}{3} \sum_{j=1}^{M} t_{j}^{2} \int_{D_{y}^{j}}\left(\beta \partial_{y}^{2} q_{2}\left(\mathbf{G}_{1}^{h}\right)_{2}-\partial_{y} \beta \partial_{y} q_{1}\left(\mathbf{G}_{1}^{h}\right)_{1}\right) d x d y-R\left(\mathbf{G}_{1}^{h}\right),
\end{aligned}
$$

where

$$
\left|R\left(\mathbf{G}_{1}^{h}\right)\right| \leq C h^{3}\|\mathbf{q}\|_{3, p}\left\|\mathbf{G}_{1}^{h}\right\|_{0, p^{\prime}}
$$

By using (4.3) and (4.5) we rewrite (4.7) as follows:

$$
\begin{aligned}
u^{h}\left(x_{i}, y_{j}\right)= & P_{h}^{0} u\left(x_{i}, y_{j}\right)+\sum_{i=1}^{N} h_{i}^{2} \int_{D_{x}^{i}} D_{1} \mathbf{q} \cdot \mathbf{G}_{1}^{h} d x d y \\
& +\sum_{j=1}^{M} t_{j}^{2} \int_{D_{y}^{j}} D_{2} \mathbf{q} \cdot \mathbf{G}_{1}^{h} d x d y-R\left(\mathbf{G}_{1}^{h}\right)
\end{aligned}
$$

with

$$
\left|R\left(\mathbf{G}_{1}^{h}\right)\right| \leq C h^{2+\frac{2}{p}}|\log h|^{\frac{1}{2}}\|\mathbf{q}\|_{3, p}, \quad 1<p \leq 2 ;
$$

here we have used (A.5) to estimate $\left\|\mathbf{G}_{1}^{h}\right\|_{0, p^{\prime}}$ in (4.8). To get the relation (4.1), replace $\mathbf{G}_{1}^{h}$ by $\mathbf{G}_{1}$ in (4.9) and let $E_{1}^{h}\left(x_{i}, y_{j}\right)$ denote the collection of remainders and other terms. Clearly, it suffices to estimate $E_{1}^{h}\left(x_{i}, y_{j}\right)$. By the Hölder inequality we have

$$
\left|E_{1}^{h}\left(x_{i}, y_{j}\right)\right| \leq C h^{2}\left\|\mathbf{G}_{1}-\mathbf{G}_{1}^{h}\right\|_{0, s^{\prime}}\|\mathbf{q}\|_{2, s}+\left|R\left(\mathbf{G}_{1}^{h}\right)\right|,
$$

where $s=\frac{2 p}{2-p}$ and $s^{\prime}$ is the conjugate of $s$.

Thus, it follows from (A.3), (A.4), and the Sobolev inequality that

$$
\left\|\mathbf{G}_{1}-\mathbf{G}_{1}^{h}\right\|_{0, s^{\prime}}\|\mathbf{q}\|_{2, s} \leq \begin{cases}C h \log \frac{1}{h}\|\mathbf{q}\|_{2, \infty}, & p=2, \\ C\left(C(p)+\log \frac{1}{h}\right) h^{\frac{2}{p^{\prime}}}\|\mathbf{q}\|_{3, p}, & 1<p<2 .\end{cases}
$$

Now, combining (4.11) with (4.12) and (4.10) yields (4.2).

The relation (4.1) along with (4.2) is actually the asymptotic error expansion desired for the approximation of the scalar field. It should be noted that $u^{h}$ was 
expanded with respect to $P_{h}^{0} u$ rather than the function $u$ itself. We are then forced to take appropriate refinements in order to carry out the Richardson extrapolation process for this problem. This section will be devoted to an analysis of a procedure that uses less expensive refinements. The procedure is effectively a combination of extrapolation with local postprocessing. In other words, we shall derive a better approximation by using the superconvergence results for the vector field along with the technique of extrapolation for the scalar field. A procedure of pure extrapolation for the approximate scalar field will be considered later in $\S 5$, where different refined grids are needed. But we emphasize that all the results for the scalar field are valid for nonuniform grids, since the Green's function $\left(\mathbf{G}_{1}, \lambda_{1}\right)$ has less singularity in comparison with $\left(\mathbf{G}_{2}, \lambda_{2}\right)$.

Let $\Delta_{2, x}$ and $\Delta_{2, y}$ be two partitions defined by

$$
\Delta_{2, x}: \quad 0=x_{0}^{*}<x_{1}<x_{1}^{*}<x_{2}<x_{2}^{*}<\cdots<x_{N}<x_{N}^{*}=1
$$

and

$$
\Delta_{2, y}: \quad 0=y_{0}^{*}<y_{1}<y_{1}^{*}<y_{2}<y_{2}^{*}<\cdots<y_{M}<y_{M}^{*}=1,
$$

where $x_{i}, y_{j}$ are the centers of $I_{x}^{i}$ and $I_{y}^{j}$, respectively.

Denote by $\Re_{1, h}$ and $\Re_{2, h}$ the two refinements for $\Re_{h}$ given by $\Delta_{2, x} \times \Delta_{y}$ and $\Delta_{x} \times \Delta_{2, y}$, respectively. Let $\mathbf{V}_{i}^{h} \times M_{i}^{h}$ be the RT space of the lowest order associated with the partition $\Re_{i, h}$, and let $\Pi_{i, h}$ and $P_{i, h}^{0}, i=1,2$, be the corresponding projection operators. Let $\left(\mathbf{q}^{i, h}, u^{i, h}\right)$ be the mixed finite element approximations of (1.3) lying in $\mathbf{V}_{i}^{h} \times M_{i}^{h}$ for $i=1,2$.

For any $\left(x_{i}, y_{j}\right)$, center of the element $I_{x}^{i} \times I_{y}^{j} \in \Re_{h}$, denote by $\left(\mathbf{G}_{1}^{\kappa, h}, \lambda_{1}^{\kappa, h}\right)$ the mixed finite element approximations of the regularized Green's function $\left(\mathbf{G}_{1}, \lambda_{1}\right)$ at $\left(x_{i}, y_{j}\right)$ in the spaces $\mathbf{V}_{\kappa}^{h} \times M_{\kappa}^{h}, \kappa=1,2$, respectively. The following analogue of (A.3) still holds:

$$
\sum_{\kappa=1}^{2}\left\|\mathbf{G}_{1}^{\kappa, h}-\mathbf{G}_{1}\right\|_{0,1} \leq C h \log \frac{1}{h} .
$$

The proof of (4.13) can be accomplished by employing the same arguments as those used to prove (A.3).

For any $R=I_{x}^{i} \times I_{y}^{j} \in \Re_{h}$, there exist two elements $R_{\kappa 1}$ and $R_{\kappa 2}$ in $\Re_{\kappa, h}$ such that $R=R_{\kappa 1} \cup R_{\kappa 2}$ (see Figure 2).

Set

$$
\Xi_{h}=\left\{\left(x_{i}, y_{j}\right) ; \quad 1 \leq i \leq N, 1 \leq j \leq M\right\} ;
$$

i.e., $\Xi_{h}$ is the collection of centers of gravity of elements in $\Re_{h}$. Let us define a new approximation, denoted by $u_{h}^{\#}$, on $\Xi_{h}$ to the scalar field $u$ by

$$
\begin{aligned}
u_{h}^{\#}\left(x_{i}, y_{j}\right)= & \frac{1}{3}\left(4\left(\bar{u}^{1, h}+\bar{u}^{2, h}\right)-5 u^{h}\right)\left(x_{i}, y_{j}\right) \\
& +\frac{1}{6}\left(h_{i}^{2} \partial_{x}\left(\beta q_{1}^{h}\right)+t_{j}^{2} \partial_{y}\left(\beta q_{2}^{h}\right)\right)\left(x_{i}, y_{j}\right),
\end{aligned}
$$




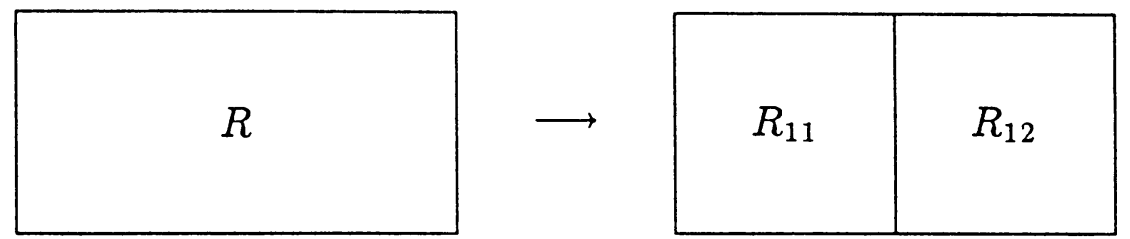

FIGURE 2

Illustration of refinement in the $x$-direction

where

$$
\bar{u}^{\kappa, h}=\frac{1}{2}\left(\left.u^{\kappa, h}\right|_{R_{\kappa 1}}+\left.u^{\kappa, h}\right|_{R_{\kappa 2}}\right), \quad \kappa=1,2 .
$$

Theorem 4.1. Let $u_{h}^{\#}$ be defined on $\Xi_{h}$ by (4.14). Let $p$ be any number in $(1,2]$ and $p^{\prime}$ its conjugate number. Then

$$
\left\|u-u_{h}^{\#}\right\|_{0, \infty, \Xi_{h}} \leq \begin{cases}C h^{3} \log \frac{1}{h}\left(\|\mathbf{q}\|_{2, \infty}+\|\mathbf{q}\|_{3,2}\right), & p=2, \\ C(p) h^{2+\frac{2}{p^{\prime}} \log \frac{1}{h}\|\mathbf{q}\|_{3, p},} & 1<p<2,\end{cases}
$$

provided that $\mathbf{q} \in\left[W^{3, p}\right]^{2}$ for $p<2$ and $\mathbf{q} \in\left[W^{3,2}\right]^{2} \cap\left[W^{2, \infty}\right]^{2}$ for $p=2$.

Proof. For any $\left(x_{i}, y_{j}\right) \in \Xi_{h}$, let $R \in \Re_{h}$ be the element centered at $\left(x_{i}, y_{j}\right)$. It follows from Lemma 4.1 that the approximate solution $u^{h}$ can be expanded at $\left(x_{i}, y_{j}\right)$ with respect to $P_{h}^{0} u$ in the form (4.1). Let us derive an expansion for the average of the approximate scalar field $u^{l, h}$ analogous to (4.1). By the definition of $\delta_{1}^{h}$,

$$
\vec{u}^{l, h}\left(x_{i}, y_{j}\right)-P_{h}^{0} u\left(x_{i}, y_{j}\right)=\left(u^{l, h}-P_{l, h}^{0} u, \delta_{1}^{h}\right), \quad l=1,2 .
$$

Thus, by (4.16) and (A.10),

$$
\bar{u}^{l, h}\left(x_{i}, y_{j}\right)=P_{h}^{0} u\left(x_{i}, y_{j}\right)+\left(\beta\left(\Pi_{l, h} \mathbf{q}-\mathbf{q}\right), \mathbf{G}_{1}^{l, h}\right), \quad l=1,2 .
$$

The relation (4.17) for $\bar{u}^{l, h}, l=1,2$, is actually an analogue of (4.6) for $u^{h}$. Thus, by applying arguments similar to those used to prove (4.1), we obtain

$$
\begin{aligned}
\frac{l}{u}, h\left(x_{i}, y_{j}\right)= & P_{h}^{0} u\left(x_{i}, y_{j}\right)+\frac{3 l-2}{4} \sum_{i=1}^{N} h_{i}^{2} \int_{D_{x}^{i}} D_{1} \mathbf{q} \cdot \mathbf{G}_{1} d x d y \\
& +\frac{7-3 !}{4} \sum_{j=1}^{M} t_{j}^{2} \int_{D_{y}^{j}} D_{2} \mathbf{q} \cdot \mathbf{G}_{1} d x d y \\
& +E_{l, 1}^{h}\left(x_{i}, y_{j}\right), \quad l=1,2,
\end{aligned}
$$

where $E_{l, 1}^{h}, l=1,2$, are the corresponding remainders, which satisfy

$$
\left|E_{l, 1}^{h}\left(x_{i}, y_{j}\right)\right| \leq \begin{cases}C h^{3} \log \frac{1}{h}\left(\|\mathbf{q}\|_{2, \infty}+\|\mathbf{q}\|_{3,2}\right), & p=2, \\ C(p) h^{2+\frac{2}{p}} \log \frac{1}{h}\|\mathbf{q}\|_{3, p}, & 1<p<2 .\end{cases}
$$


Thus, it follows from (4.14) together with (4.1) and (4.18) that there exists a function $E_{2}^{h}$ such that

$$
\begin{aligned}
u_{h}^{*}\left(x_{i}, y_{j}\right)= & P_{h}^{0} u\left(x_{i}, y_{j}\right)+\frac{1}{6}\left(h_{i}^{2} \partial_{x}\left(\beta q_{1}^{h}\right)+t_{j}^{2} \partial_{y}\left(\beta q_{2}^{h}\right)\right)\left(x_{i}, y_{j}\right) \\
& +E_{2}^{h}\left(x_{i}, y_{j}\right)
\end{aligned}
$$

where

$$
\left|E_{2}^{h}\left(x_{i}, y_{j}\right)\right| \leq \begin{cases}C h^{3} \log \frac{1}{h}\left(\|\mathbf{q}\|_{2, \infty}+\|\mathbf{q}\|_{3,2}\right), & p=2, \\ C(p) h^{2+\frac{2}{p^{\prime}}} \log \frac{1}{h}\|\mathbf{q}\|_{3, p}, & 1<p<2 .\end{cases}
$$

Further, by the superconvergence result (3.18) we can find a function $E_{3}^{h}$ such that

$$
\begin{aligned}
& \left(h_{i}^{2} \partial_{x}\left(\beta q_{1}^{h}\right)+t_{j}^{2} \partial_{y}\left(\beta q_{2}^{h}\right)\right)\left(x_{i}, y_{j}\right), \\
& \quad=\left(h_{i}^{2} \partial_{x}\left(\beta q_{1}\right)+t_{j}^{2} \partial_{y}\left(\beta q_{2}\right)\right)\left(x_{i}, y_{j}\right)+E_{3}^{h}\left(x_{i}, y_{j}\right) \\
& \quad=-\left(h_{i}^{2} \partial_{x}^{2} u\left(x_{i}, y_{j}\right)+t_{j}^{2} \partial_{y}^{2} u\left(x_{i}, y_{j}\right)\right)+E_{3}^{h}\left(x_{i}, y_{j}\right),
\end{aligned}
$$

where, by the Sobolev inequality,

$$
\begin{aligned}
\left|E_{3}^{h}\left(x_{i}, y_{j}\right)\right| & \leq \begin{cases}C h^{3} \log \frac{1}{h}\|\mathbf{q}\|_{2, \infty}, & p=2, \\
C h^{2+\frac{2}{p}} \log \frac{1}{h}\|\mathbf{q}\|_{2, \frac{2 p}{2-p}}, & 1<p<2 .\end{cases} \\
& \leq \begin{cases}C h^{3} \log \frac{1}{h}\|\mathbf{q}\|_{2, \infty}, & p=2, \\
C(p) h^{2+\frac{2}{p^{t}}} \log \frac{1}{h}\|\mathbf{q}\|_{3, p}, & 1<p<2 .\end{cases}
\end{aligned}
$$

Thus, combining (4.20) with (4.21) gives

$$
u_{h}^{\#}\left(x_{i}, y_{j}\right)=P_{h}^{0} u\left(x_{i}, y_{j}\right)-\frac{1}{6}\left(h_{i}^{2} \partial_{x}^{2} u+t_{j}^{2} \partial_{y}^{2} u\right)\left(x_{i}, y_{j}\right)+E^{h}\left(x_{i}, y_{j}\right),
$$

where the remainder $E^{h}$ can be bounded by the right-hand side of (4.2).

It is not difficult to verify that

$$
P_{h}^{0} u\left(x_{i}, y_{j}\right)=u\left(x_{i}, y_{j}\right)+\frac{1}{6}\left(h_{i}^{2} \partial_{x}^{2} u+t_{j}^{2} \partial_{y}^{2} u\right)\left(x_{i}, y_{j}\right)+O\left(h^{2+\frac{2}{p}}\right)\|u\|_{3, p} .
$$

Hence, (4.15) follows from (4.23) and (4.24).

Remark 4.1. It is clear from the definition of the regularized Green's function that the so-called regularized Green's functions contain the mesh size $h$ as a parameter. However, this dependence does not affect the estimate in the extrapolation, since we can always choose the same regularized Dirac $\delta$-function for the approximations used in the extrapolation. One may want to use the true Green's function in doing the expansion rather than the regularized one. The difficulty of doing so is that one has to deal with the singularity caused by the $\delta$-function. Doing the estimates for the regularized Green's function is much easier than for the true Green's function. This idea can be applied to the expansion for the standard Galerkin approximation for the Laplace equation. This remark is also applicable to the vector field in $\S 5$. 
As in [20] and [3], a local postprocessing procedure can be applied to produce an approximation defined globally, which has the same accuracy as $u_{h}^{\#}$ on $\Xi_{h}$. This result is stated as follows. For any $\mathbf{x} \in R=I_{x}^{i} \times I_{y}^{j}$, let $\overline{\mathbf{x}}=\left(x_{i}, y_{j}\right)$ be the center of gravity of $R$. Define $u_{h}^{\#}(\mathbf{x})$ by

$$
\begin{aligned}
u_{h}^{\#}(\mathbf{x})= & u_{h}^{\#}(\overline{\mathbf{x}})-\beta(\overline{\mathbf{x}}) \mathbf{q}^{h}(\overline{\mathbf{x}}) \cdot(\mathbf{x}-\overline{\mathbf{x}}) \\
& -\frac{1}{2}(\mathbf{x}-\overline{\mathbf{x}}) \nabla\left(\beta \mathbf{q}^{h}\right)(\overline{\mathbf{x}})(\mathbf{x}-\overline{\mathbf{x}})^{t}, \quad \mathbf{x} \in R,
\end{aligned}
$$

where $\boldsymbol{\psi}^{t}$ indicates the transpose of the vector $\boldsymbol{\psi}$.

Theorem 4.2. Under the assumptions of Theorem 4.1,

$$
\left\|u_{h}^{\#}-u\right\|_{0, \infty} \leq \begin{cases}C h^{3} \log \frac{1}{h}\left(\|\mathbf{q}\|_{2, \infty}+\|\mathbf{q}\|_{3,2}\right), & p=2, \\ C(p) h^{2+\frac{2}{p}} \log \frac{1}{h}\|\mathbf{q}\|_{3, p}, & 1<p<2,\end{cases}
$$

where $p^{\prime}=p /(p-1)$.

The superconvergence result of Theorem 3.2 plays an important role in deriving (4.26). As the proof of Theorem 4.2 is quite simple, we omit the details.

\section{EXTRAPOLATION FOR THE VELOCITY}

It is well known that the technique of extrapolation is contingent upon the existence of an adequate asymptotic error expansion for the approximation considered. Therefore, we begin with the derivation of an asymptotic error expansion for the RT approximation of the vector field.

Let $\Re_{h}=\Delta_{x} \times \Delta_{y}$ be the rectangular partition of $\Omega$. We shall assume throughout this section that $\Delta_{x}$ and $\Delta_{y}$ are two uniform partitions of $(0,1)$. In order to describe two sets of points on which the approximate vector field can be extrapolated, let $\widehat{R}=(-1,1) \times(-1,1)$ be a reference element. Introduce two sets of points on $\widehat{R}$ by setting

$$
\widehat{\Xi}_{1}=\left\{(-1,0),\left(-\frac{1}{2}, 0\right),(0,0),\left(\frac{1}{2}, 0\right),(1,0)\right\}
$$

and

$$
\widehat{\Xi}_{2}=\left\{(0,-1),\left(0,-\frac{1}{2}\right),(0,0),\left(0, \frac{1}{2}\right),(0,1)\right\} .
$$

For each element $R=I_{x}^{i} \times I_{y}^{j} \in \Re_{h}$, let $F$ be the affine mapping

$$
F: \hat{R} \longrightarrow R \text {. }
$$

Then we can introduce two sets of points on each elemerit by setting

$$
\Xi_{1, h}^{i, j}=F\left(\hat{\Xi}_{1}\right) \text { and } \Xi_{2, h}^{i, j}=F\left(\hat{\Xi}_{2}\right) \text {. }
$$

Let $\Xi_{1, h}$ and $\Xi_{2, h}$ be the collection of $\Xi_{1, h}^{i, j}$ and $\Xi_{2, h}^{i, j}$, respectively; i.e.,

$$
\Xi_{1, h}=\left\{\Xi_{1, h}^{i, j} ; \quad 1 \leq i \leq N, 1 \leq j \leq M\right\}
$$

and

$$
\Xi_{2, h}=\left\{\Xi_{2, h}^{i, j} ; \quad 1 \leq i \leq N, 1 \leq j \leq M\right\} .
$$


We shall extrapolate the approximations of two components of the vector field on the sets $\Xi_{1, h}$ and $\Xi_{2, h}$, respectively. For the sake of simplicity of analysis, we shall confine ourselves to the treatment of the first component of the vector field, as the analysis of the second component is similar.

Lemma 5.1. Let $\left(\mathbf{q}^{h}, u^{h}\right)$ be the mixed finite element approximation of (1.3) in the RT space of the lowest order. Let $\mathbf{q} \in\left[W^{3, p}(\Omega)\right]^{2}$ with $1 \leq p \leq \infty$. Then,

$$
q_{1}^{h}(\mathbf{x})=\Pi_{h} q_{1}(\mathbf{x})-h_{1}^{2} \int_{\Omega} D_{1} \mathbf{q} \cdot \mathbf{G}_{2}^{h} d x d y-t_{1}^{2} \int_{\Omega} D_{2} \mathbf{q} \cdot \mathbf{G}_{2}^{h} d x d y+R_{1}^{h}(\mathbf{x})
$$

with

$$
\left\|R_{1}^{h}\right\|_{0, \infty} \leq \begin{cases}C h^{3-\frac{2}{p}} \log \frac{1}{h}\|\mathbf{q}\|_{3, p}, & 2<p \leq \infty, \\ C h^{3-\frac{2}{p}}\|\mathbf{q}\|_{3, p}, & 1 \leq p \leq 2,\end{cases}
$$

where $D_{1} q$ and $D_{2} \mathbf{q}$ are two vector-valued functions defined by (4.3) and (4.4), respectively.

Remark 5.1. Since the partitions $\Delta_{x}$ and $\Delta_{y}$ are uniform, we can denote by $h_{1}$ and $t_{1}$ the mesh sizes in the $x$ - and $y$-directions, respectively. Moreover, the terms of second order in the expansion can be represented as single integrals rather than sums of integrals over elements.

Proof of Lemma 5.1. For any point $\mathbf{x} \in \Omega$, it is easy to see that

$$
q_{1}^{h}(\mathbf{x})-\Pi_{h} q_{1}(\mathbf{x})=\left(\mathbf{q}^{h}-\Pi_{h} \mathbf{q}, \boldsymbol{\delta}_{2}^{h}\right),
$$

where $\delta_{2}^{h}$ is a regularized Dirac $\delta$-function at $\mathbf{x}$. Let $\left(\mathbf{G}_{2}^{h}, \lambda_{2}^{h}\right)$ be the mixed finite element approximation of the regularized Green's function $\left(\mathbf{G}_{2}, \lambda_{2}\right)$ associated with $\delta_{2}^{h}$. By (A.11),

$$
\left(\mathbf{q}^{h}-\Pi_{h} \mathbf{q}, \boldsymbol{\delta}_{2}^{h}\right)=\left(\beta\left(\mathbf{q}-\Pi_{h} \mathbf{q}\right), \mathbf{G}_{2}^{h}\right) .
$$

It follows from (5.3) and (5.4) that

$$
q_{1}^{h}(\mathbf{x})-\Pi_{h} q_{1}(\mathbf{x})=\left(\beta\left(\mathbf{q}-\Pi_{h} \mathbf{q}\right), \mathbf{G}_{2}^{h}\right) .
$$

Thus, applying (2.15), with $\mathbf{v}$ replaced by $\mathbf{G}_{2}^{h}$, leads to

$$
q_{1}^{h}(\mathbf{x})-\Pi_{h} q_{1}(\mathbf{x})=-h_{1}^{2} \int_{\Omega} D_{1}^{2} \mathbf{q} \cdot \mathbf{G}_{2}^{h} d x d y-t_{1}^{2} \int_{\Omega} D_{2}^{2} \mathbf{q} \cdot \mathbf{G}_{2}^{h} d x d y+R_{1}^{h}(\mathbf{x})
$$

with

$$
\left|R_{1}^{h}(\mathbf{x})\right| \leq C h^{3}\|\mathbf{q}\|_{3, p}\left\|\mathbf{G}_{2}^{h}\right\|_{0, p^{\prime}}
$$

where $D_{1} q$ and $D_{2} q$ are two vector-valued functions defined in (4.3) and (4.4), respectively. To estimate (5.7), we apply (A.9) to obtain

$$
\left|R_{1}^{h}(\mathbf{x})\right| \leq \begin{cases}C h^{3-\frac{2}{p}} \log \frac{1}{h}\|\mathbf{q}\|_{3, p}, & 2<p \leq \infty, \\ C h^{3-\frac{2}{p}}\|\mathbf{q}\|_{3, p}, & 1 \leq p \leq 2 .\end{cases}
$$

Thus, it follows from (5.6) and (5.8) that (5.1) and (5.2) hold.

If we restrict the point $\mathbf{x}$ to vary along the Gauss lines in the $x$-direction, we shall obtain an asymptotic error expansion of the following form. 
Lemma 5.2. Let $\mathbf{q} \in\left[W^{3, p}(\Omega)\right]^{2}$ with $2<p<\infty$. Then for any $\left(x, y_{j}\right) \in$ $I_{x}^{i} \times I_{y}^{j}$, there exists a function $R_{2}^{h}$ such that

$$
\begin{aligned}
q_{1}^{h}\left(x, y_{j}\right)= & q_{1}\left(x, y_{j}\right)+\frac{1}{2}\left[h_{1}^{2}-\left(x-x_{i}\right)^{2}\right] \partial_{x}^{2} q_{1}\left(x, y_{j}\right)+\frac{t_{1}^{2}}{6} \partial_{y}^{2} q_{1}\left(x, y_{j}\right) \\
& -h_{1}^{2} \int_{\Omega} D_{1} \mathbf{q} \cdot \mathbf{G}_{2} d x d y-t_{1}^{2} \int_{\Omega} D_{2} \mathbf{q} \cdot \mathbf{G}_{2} d x d y+R_{2}^{h}\left(x, y_{j}\right)
\end{aligned}
$$

with

$$
\left|R_{2}^{h}\left(x, y_{j}\right)\right| \leq C\left(C(p)+\log \frac{1}{h}\right) h^{3-\frac{2}{p}}\|\mathbf{q}\|_{3, p} .
$$

Proof. Since the point $\left(x, y_{j}\right)$ lies on the Gauss lines of the element $I_{x}^{i} \times I_{y}^{j}$, it follows from the assumption and the expansion (2.11) that

$$
\begin{aligned}
\Pi_{h} q_{1}\left(x, y_{j}\right)= & q_{1}\left(x, y_{j}\right)+\frac{1}{2}\left[h_{1}^{2}-\left(x-x_{i}\right)^{2}\right] \partial_{x}^{2} q_{1}\left(x, y_{j}\right) \\
& +\frac{t_{1}^{2}}{6} \partial_{y}^{2} q_{1}\left(x, y_{j}\right)+O\left(h^{3-\frac{2}{p}}\right)\|\mathbf{q}\|_{3, p} .
\end{aligned}
$$

Here, $h_{1}$ and $t_{1}$ represent the mesh sizes in the $x$ - and $y$-directions, respectively. Note that the partition is uniform in both directions. Thus, combining (5.1) with (5.11) yields

$$
\begin{aligned}
q_{1}^{h}\left(x, y_{j}\right)= & q_{1}\left(x, y_{j}\right)+\frac{1}{2}\left[h_{1}^{2}-\left(x-x_{i}\right)^{2}\right] \partial_{x}^{2} q_{1}\left(x, y_{j}\right)+\frac{t_{1}^{2}}{6} \partial_{y}^{2} q_{1}\left(x, y_{j}\right) \\
& -h_{1}^{2} \int_{\Omega} D_{1} \mathbf{q} \cdot \mathbf{G}_{2}^{h} d x d y-t_{1}^{2} \int_{\Omega} D_{2} \mathbf{q} \cdot \mathbf{G}_{2}^{h} d x d y+R_{3}^{h}\left(x, y_{j}\right),
\end{aligned}
$$

where

$$
R_{3}^{h}\left(x, y_{j}\right)=R_{2}^{h}\left(x, y_{j}\right)+O\left(h^{3-\frac{2}{p}}\right)\|\mathbf{q}\|_{3, p} .
$$

To deal with the integral terms in the right-hand side of (5.12), we observe that

$$
\int_{\Omega} D_{1} \mathbf{q} \cdot \mathbf{G}_{2}^{h} d x d y=\int_{\Omega} D_{1} \mathbf{q} \cdot \mathbf{G}_{2} d x d y+\int_{\Omega} D_{1} \mathbf{q} \cdot\left(\mathbf{G}_{2}^{h}-\mathbf{G}_{2}\right) d x d y .
$$

Thus, by (5.23) below,

$$
\int_{\Omega} D_{1} \mathbf{q} \cdot \mathbf{G}_{2}^{h} d x d y=\int_{\Omega} D_{1} \mathbf{q} \cdot \mathbf{G}_{2} d x d y+O\left(h^{1-\frac{2}{p}}\right)\left(C(p)+|\log h|^{\frac{1}{2}}\right)\|\mathbf{q}\|_{3, p} .
$$

Similarly,

$$
\int_{\Omega} D_{2} \mathbf{q} \cdot \mathbf{G}_{2}^{h} d x d y=\int_{\Omega} D_{2} \mathbf{q} \cdot \mathbf{G}_{2} d x d y+O\left(h^{1-\frac{2}{p}}\right)\left(C(p)+|\log h|^{\frac{1}{2}}\right)\|\mathbf{q}\|_{3, p} .
$$

Thus, it follows from (5.12) together with (5.14) and (5.15) that there exists $R_{2}^{h}\left(x, y_{j}\right)$ satisfying (5.9) and (5.10).

The relation (5.9) is in fact the asymptotic error expansion for the approximation of the first component of the vector field. It should be noted that the 

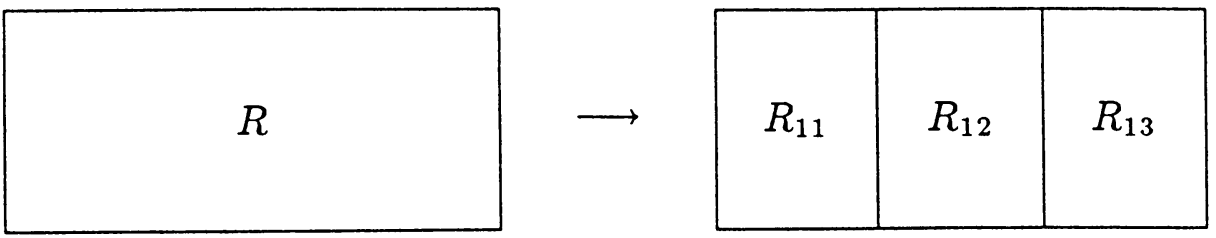

Figure 3

Illustration of refinement in the $x$-direction

point $\left(x, y_{j}\right)$ can vary along the Gauss lines in the $x$-direction. But we shall be interested in those points on which the terms of second order in the expansion (5.9) can be extrapolated out. It will be seen that these points form the set $\Xi_{1, h}$.

Before presenting any procedure of extrapolation for the vector field, we describe two different refinements for the original partition $\Re_{h}=\Delta_{x} \times \Delta_{y}$. Let us subdivide each interval of $\Delta_{x}$ into three equally sized subintervals, and denote by $\Delta_{3, x}$ the corresponding refinement of $\Delta_{x}$. Then a refinement of $\Re_{h}$ can be defined to be $\Re_{3, h}=\Delta_{3, x} \times \Delta_{y}$. Similarly, if $\Delta_{3, y}$ is an analogous refinement for $\Delta_{y}$, then $\Re_{4, h}=\Delta_{x} \times \Delta_{3, y}$ can be defined as another refinement to $\Re_{h}$. Let $R$ be any element in $\Re_{h}$; then there must be three elements $R_{\kappa 1}, R_{\kappa 2}$, and $R_{\kappa 3}$ in $\Re_{2+\kappa, h}$ such that $R=R_{\kappa 1} \cup R_{\kappa 2} \cup R_{\kappa 3}, \kappa=1,2$ (see Figure 3).

Let $\mathbf{V}_{\kappa}^{h} \times M_{\kappa}^{h}$ be the RT spaces of the lowest order associated with the partitions $\Re_{\kappa, h}, \kappa=3,4$, respectively. Denote by $\left(\mathbf{q}^{\kappa, h}, u^{\kappa, h}\right)$ the corresponding finite element approximations of (1.3) lying in $\mathbf{V}_{\kappa}^{h} \times M_{\kappa}^{h}, \kappa=3$, 4. An extrapolation procedure for the first component of the vector field can be described as follows. Let $\mathbf{x} \in \Xi_{1, h}$, and define a new approximate solution to $q_{1}(\mathbf{x})$ by

$$
q_{1}^{\#}(\mathbf{x})=\frac{1}{8}\left(9\left(q_{1}^{3, h}+q_{1}^{4, h}\right)-10 q_{1}^{h}\right)(\mathbf{x}) .
$$

The following theorem provides an error estimate for this new approximation.

Theorem 5.1. Let $q_{1}^{\#}$ be defined on $\Xi_{1, h}$ by (5.16), and assume that $\mathbf{q} \in$ $\left[W^{3, p}(\Omega)\right]^{2}$ with $2<p<\infty$. Then

$$
\left\|q_{1}-q_{1}^{\#}\right\|_{0, \infty, \Xi_{1, h}} \leq C\left(C(p)+\log \frac{1}{h}\right) h^{3-\frac{2}{p}}\|\mathbf{q}\|_{3, p} .
$$

Proof. Since any point $\mathbf{x} \in \Xi_{1, h}$ is on the Gauss lines of the partitions $\Re_{h}$, $\Re_{3, h}$, and $\Re_{4, h}$, we can expand $q_{1}^{h}(\mathbf{x}), q_{1}^{3, h}(\mathbf{x})$ and $q_{1}^{4, h}(\mathbf{x})$ in terms of $q_{1}(\mathbf{x})$ and powers of the corresponding mesh sizes. The main purpose of the procedure (5.16) is to eliminate the terms of second order from the asymptotic expansion for the error. More precisely, let $\mathbf{x}$ be any point in $\Xi_{1, h}$. According to the definition of the set $\Xi_{1, h}$, we can have the following configurations for $\mathbf{x}$ :

(1) $\mathbf{x}=F[(0,0)]$,

(2) $\mathbf{x}=F[(-1,0)]$ or $F[(1,0)]$, 
(3) $\mathbf{x}=F\left[\left(-\frac{1}{2}, 0\right)\right]$ or $F\left[\left(\frac{1}{2}, 0\right)\right]$.

Here, $F$ is the affine mapping from the reference element onto elements in $\Re_{h}$.

Let us deal with the first case; the second and third can be analyzed similarly. Thus, the point $\mathbf{x}$ is the center of an element in $\Re_{h}$. Assume, without loss of generality, that $\mathbf{x}=\left(x_{i}, y_{j}\right)$ is the center of the element $I_{x}^{i} \times I_{y}^{j} \in \Re_{h}$. Applying the asymptotic error expansion (5.9) at $\left(x_{i}, y_{j}\right)$ to $q_{1}^{h}, q_{1}^{3, h}$, and $q_{1}^{4, h}$, respectively, yields

and

$$
\begin{aligned}
q_{1}^{h}\left(x_{i}, y_{j}\right)= & q_{1}\left(x_{i}, y_{j}\right)+\frac{h_{1}^{2}}{2} \partial_{x}^{2} q_{1}\left(x_{i}, y_{j}\right)+\frac{t_{1}^{2}}{6} \partial_{y}^{2} q_{1}\left(x_{i}, y_{j}\right) \\
& -h_{1}^{2} \int_{\Omega} D_{1} \mathbf{q} \cdot \mathbf{G}_{2} d x d y-t_{1}^{2} \int_{\Omega} D_{2} \mathbf{q} \cdot \mathbf{G}_{2} d x d y \\
& +R_{2}^{h}\left(x_{i}, y_{j}\right), \\
q_{1}^{3, h}\left(x_{i}, y_{j}\right)= & q_{1}\left(x_{i}, y_{j}\right)+\frac{h_{1}^{2}}{18} \partial_{x}^{2} q_{1}\left(x_{i}, y_{j}\right)+\frac{t_{1}^{2}}{6} \partial_{y}^{2} q_{1}\left(x_{i}, y_{j}\right) \\
& -\frac{h_{1}^{2}}{9} \int_{\Omega} D_{1} \mathbf{q} \cdot \mathbf{G}_{2} d x d y-t_{1}^{2} \int_{\Omega} D_{2} \mathbf{q} \cdot \mathbf{G}_{2} d x d y \\
& +R_{2}^{3, h}\left(x_{i}, y_{j}\right),
\end{aligned}
$$

$$
\begin{aligned}
q_{1}^{4, h}\left(x_{i}, y_{j}\right)= & q_{1}\left(x_{i}, y_{j}\right)+\frac{h_{1}^{2}}{2} \partial_{x}^{2} q_{1}\left(x_{i}, y_{j}\right)+\frac{t_{1}^{2}}{54} \partial_{y}^{2} q_{1}\left(x_{i}, y_{j}\right) \\
& -h_{1}^{2} \int_{\Omega} D_{1} \mathbf{q} \cdot \mathbf{G}_{2} d x d y-\frac{t_{1}^{2}}{9} \int_{\Omega} D_{2} \mathbf{q} \cdot \mathbf{G}_{2} d x d y \\
& +R_{2}^{4, h}\left(x_{i}, y_{j}\right),
\end{aligned}
$$

where $R_{2}^{3, h}$ and $R_{2}^{4, h}$ are the corresponding remainders. It is obvious that their maximum norms can be bounded uniformly from above by the right-hand side of (5.10). Thus, combining (5.16) with the relations (5.18), (5.19), and (5.20) yields

$$
\left|q_{1}^{\#}\left(x_{i}, y_{j}\right)-q_{1}\left(x_{i}, y_{j}\right)\right| \leq C\left(C(p)+\log \frac{1}{h}\right) h^{3-\frac{2}{p}}\|\mathbf{q}\|_{3, p},
$$

which establishes Theorem 5.1 in the case (1).

A similar result can be derived for the second component of the approximate vector field. To do so, let us define a new approximation to $q_{2}$ on the set $\Xi_{2, h}$ by setting

$$
q_{2}^{\#}(x, y)=\frac{1}{8}\left(9\left(q_{2}^{3, h}+q_{2}^{4, h}\right)-10 q_{2}^{h}\right)(\mathbf{x}), \quad \mathbf{x} \in \Xi_{2, h} .
$$

Theorem 5.2. Let $q_{2}^{\#}$ be defined on $\Xi_{2, h}$ by (5.21), and assume that $\mathbf{q} \in$ $\left[W^{3, p}(\Omega)\right]^{2}$ with $2<p<\infty$. Then

$$
\left\|q_{2}-q_{2}^{\#}\right\|_{0, \infty, \Xi_{2, h}} \leq C\left(C(p)+\log \frac{1}{h}\right) h^{3-\frac{2}{p}}\|\mathbf{q}\|_{3, p} .
$$


To complete the proof of Theorem 5.1, we still need to establish the following lemma.

Lemma 5.3. Let $\left(\mathbf{G}_{2}^{h}, \lambda_{2}^{h}\right)$ be the mixed finite element approximation of $\left(\mathbf{G}_{2}, \lambda_{2}\right)$. Then

$$
\left|\left(\mathbf{s}, \mathbf{G}_{2}-\mathbf{G}_{2}^{h}\right)\right| \leq C\left(C(p)+|\log h|^{\frac{1}{2}}\right) h^{1-\frac{2}{p}}\|\mathbf{s}\|_{1, p},
$$

provided that $\mathbf{s} \in\left[W^{1, p}(\Omega)\right]^{2}$ with $p>2$.

Proof. Let $(\sigma, \tau)$ be a vector-valued function defined by the equations

$$
\begin{aligned}
& \beta \boldsymbol{\sigma}+\nabla \tau=\mathbf{s} \quad \text { in } \Omega, \\
& \operatorname{div} \sigma=0 \quad \text { in } \Omega, \\
& \tau=0 \quad \text { on } \partial \Omega \text {. }
\end{aligned}
$$

Let $\Pi_{h} \boldsymbol{\sigma}$ be the projection of $\boldsymbol{\sigma}$ into the finite element space $\mathbf{V}^{h}$. It is easy to see that

$$
\left(\beta \Pi_{h} \boldsymbol{\sigma}, \mathbf{G}_{2}-\mathbf{G}_{2}^{h}\right)=\left(\operatorname{div}\left(\Pi_{h} \boldsymbol{\sigma}\right), \lambda_{2}-\lambda_{2}^{h}\right)=0 .
$$

Thus, by (5.24) and the fact that $\operatorname{div} \mathbf{G}_{2}=\operatorname{div} \mathbf{G}_{2}^{h}=0$,

$$
\left(\mathbf{s}, \mathbf{G}_{2}-\mathbf{G}_{2}^{h}\right)=\left(\beta \boldsymbol{\sigma}, \mathbf{G}_{2}-\mathbf{G}_{2}^{h}\right) .
$$

Combining (5.26) with (5.25) gives

$$
\left(\mathbf{s}, \mathbf{G}_{2}-\mathbf{G}_{2}^{h}\right)=\left(\beta\left(\boldsymbol{\sigma}-\Pi_{h} \boldsymbol{\sigma}\right), \mathbf{G}_{2}-\mathbf{G}_{2}^{h}\right) .
$$

Thus,

$$
\begin{aligned}
\left|\left(\mathbf{s}, \mathbf{G}_{2}-\mathbf{G}_{2}^{h}\right)\right| & \leq C\left\|\boldsymbol{\sigma}-\Pi_{h} \boldsymbol{\sigma}\right\|_{0, p}\left\|\mathbf{G}_{2}-\mathbf{G}_{2}^{h}\right\|_{0, p^{\prime}} \\
& \leq C h\|\boldsymbol{\sigma}\|_{1, p}\left\|\mathbf{G}_{2}-\mathbf{G}_{2}^{h}\right\|_{0, p^{\prime}},
\end{aligned}
$$

where $p^{\prime}=p /(p-1)$ is the conjugate of $p$. An a priori estimate in $L^{p}$ (cf. [14]) implies that

$$
\|\boldsymbol{\sigma}\|_{1, p} \leq C(p)\|\mathbf{s}\|_{1, p} .
$$

Thus, combining (5.27) with (5.28) and the estimate (A.8) gives

$$
\left|\left(\mathbf{s}, \mathbf{G}_{2}-\mathbf{G}_{2}^{h}\right)\right| \leq C\left(C(p)+|\log h|^{\frac{1}{2}}\right) h^{1-\frac{2}{p}}\|\mathbf{s}\|_{1, p},
$$

which verifies the conclusion of the lemma.

We conclude with a result on the approximation of the scalar field. In $\S 4$, we have presented a procedure of postprocessed extrapolation for the scalar field. It is clear that the refinements given in $\S 4$ are cheaper than those stated in this section, since fewer unknowns are possible there. However, if one is interested in the refinements of this section (this is the case for extrapolating the vector field), a procedure of pure Richardson extrapolation can be obtained for the approximate scalar field. 
Define on $\Xi_{h}$ a new approximate solution to the scalar field as follows:

$$
u_{h}^{*}(\mathbf{x})=\frac{1}{8}\left(9\left(u^{3, h}+u^{4, h}\right)-10 u^{h}\right)(\mathbf{x}), \quad \mathbf{x} \in \Xi_{h},
$$

where $\Xi_{h}$ is the collection of centers of elements in $\Re_{h}$. An error estimate for this new approximate solution is given in the following theorem.

Theorem 5.3. Let $u_{h}^{*}$ be defined as in (5.29). For some real number $p$ with $1<p \leq 2$, assume that $\mathbf{q} \in\left[W^{3, p}(\Omega)\right]^{2}$ for $p<2$ and $\mathbf{q} \in\left[W^{3,2}(\Omega)\right]^{2} \cap$ $\left[W^{2, \infty}(\Omega)\right]^{2}$ for $p=2$. Then

$$
\left\|u-u_{h}^{*}\right\|_{0, \infty, \Xi_{h}} \leq \begin{cases}C h^{3} \log \frac{1}{h}\left(\|\mathbf{q}\|_{2, \infty}+\|\mathbf{q}\|_{3,2}\right), & p=2, \\ C(p) h^{2+\frac{2}{p^{7}} \log \frac{1}{h}\|\mathbf{q}\|_{3, p},} & 1<p<2,\end{cases}
$$

where $p^{\prime}=\frac{p}{p-1}$ is the conjugate of $p$.

The proof of Theorem 5.3 is a consequence of the expansion (4.1) combined with the expansion (4.24). It should be noticed that any point in $\Xi_{h}$ is also a center point of two elements in $\Re_{3, h}$ and $\Re_{4, h}$, respectively. This allows us to use the expansions (4.1) and (4.24) directly to extrapolate out the terms of second order from the expansion.

\section{APPENDIX}

To make the paper self-contained, we present here the definitions and some basic estimates for the so-called regularized Green's functions. A more detailed treatment can be found in [23].

Let $\Re_{h}$ be the given rectangular partition of the domain $\Omega$. For any point $\mathbf{x} \in \Omega$ let $R=I_{x}^{i} \times I_{y}^{j} \in \Re_{h}$ be the element containing $\mathbf{x}$. Denote by $\delta_{1}^{h}$ a function defined by

$$
\delta_{1}^{h}(\boldsymbol{\xi}, \mathbf{x})=\left\{\begin{array}{cl}
\frac{1}{4 h_{i} t_{j}}, & \boldsymbol{\xi} \in I_{x}^{i} \times I_{y}^{j}, \\
0, & \text { otherwise. }
\end{array}\right.
$$

It is obvious that the function $\delta_{1}^{h}$ is a smoothed Dirac $\delta$-function associated with the point $\mathbf{x}$. Thus, a regularized Green's function $\left(\mathbf{G}_{1}, \lambda_{1}\right)$ of the first kind can be defined as the solution of the following linear system:

$$
\begin{aligned}
\beta \mathbf{G}_{1}+\nabla \lambda_{1} & =0 & & \text { in } \Omega, \\
\operatorname{div} \mathbf{G}_{1} & =\delta_{1}^{h} & & \text { in } \Omega, \\
\lambda_{1} & =0 & & \text { on } \partial \Omega .
\end{aligned}
$$

Let $\left(\mathbf{G}_{1}^{h}, \lambda_{1}^{h}\right)$ be the mixed finite element approximation of $\left(\mathbf{G}_{1}, \lambda_{1}\right)$ using the RT methods. Then the following results can be established (cf. [23]).

Theorem 1. Let $\left(\mathbf{G}_{1}, \lambda_{1}\right)$ and $\left(\mathbf{G}_{1}^{h}, \lambda_{1}^{h}\right)$ be the solution and the mixed finite element approximation of (A.2), respectively. Let $p^{\prime}$ be any number in $(1, \infty)$. 
Then

$$
\begin{aligned}
\left\|\mathbf{G}_{1}-\mathbf{G}_{1}^{h}\right\|_{0,1} & \leq C h \log \frac{1}{h}, \\
\left\|\mathbf{G}_{1}-\mathbf{G}_{1}^{h}\right\|_{0, p^{\prime}} & \leq C\left(C(p)+\log \frac{1}{h}\right) h^{1-\frac{2}{p}}, \quad 1<p^{\prime}<\infty, \\
\left\|\mathbf{G}_{1}^{h}\right\|_{0, p^{\prime}} & \leq C h^{\min \left\{0, \frac{2}{p^{7}}-1\right\}}|\log h|^{\frac{1}{2}}, \quad 1<p^{\prime}<\infty,
\end{aligned}
$$

where $p=\frac{p^{\prime}}{p^{\prime}-1}$ is the conjugate of $p^{\prime}$.

Similarly, a second regularized Green's function can be defined by a different use of a smoothed Dirac $\delta$-function in the differential equation. This can be done as follows: Let $\delta_{2}^{h}$ be a smoothed Dirac $\delta$-function at the point $\mathbf{x}$. Define a second regularized Green's function $\left(\mathbf{G}_{2}(\boldsymbol{\xi}, \mathbf{x}), \lambda_{2}(\boldsymbol{\xi}, \mathbf{x})\right)$ as the solution of the following linear system:

$$
\begin{aligned}
\beta \mathbf{G}_{2}+\nabla \lambda_{2} & =\boldsymbol{\delta}_{2}^{h} & & \text { in } \Omega, \\
\operatorname{div} \mathbf{G}_{2} & =0 & & \text { in } \Omega, \\
\lambda_{2} & =0 & & \text { on } \partial \Omega,
\end{aligned}
$$

where $\delta_{2}^{h}=\delta_{2}^{h}(\boldsymbol{\xi}, \mathbf{x})$ is either $\left(\delta_{2}^{h}, 0\right)$ or $\left(0, \delta_{2}^{h}\right) ; \delta_{2}^{h}$ can be suitably adjusted to meet various needs of the analysis in $\S 5$. Assume also that the vector-valued function $\boldsymbol{\delta}_{2}^{h} \in \mathbf{H}$ (div) has compact support in $\Omega$. Let $\left(\mathbf{G}_{2}^{h}, \lambda_{2}^{h}\right)$ be the mixed finite element approximation of $\left(G_{2}, \lambda_{2}\right)$ in an RT space. Then the following theorem is valid.

Theorem 2. Let $p^{\prime}$ be any number in $[1, \infty]$. Then

$$
\left\|\mathbf{G}_{2}-\mathbf{G}_{2}^{h}\right\|_{0,1} \leq C|\log h|^{\frac{1}{2}} \text {, }
$$

$$
\begin{aligned}
\left\|\mathbf{G}_{2}-\mathbf{G}_{2}^{h}\right\|_{0, p^{\prime}} & \leq C\left(C(p)+|\log h|^{\frac{1}{2}}\right) h^{-\frac{2}{p}}, \quad 1<p^{\prime}<\infty, \\
\left\|\mathbf{G}_{2}^{h}\right\|_{0, p^{\prime}} & \leq \begin{cases}C h^{-\frac{2}{p}} \log \frac{1}{h}, & 1 \leq p^{\prime}<2, \\
C h^{-\frac{2}{p}}, & p^{\prime} \geq 2,\end{cases}
\end{aligned}
$$

where $p$ is the conjugate of $p^{\prime}$.

It follows from the definitions of the regularized Green's functions that the following identities are true:

$$
\left(u^{h}-P_{h}^{0} u, \delta_{1}^{h}\right)=\left(\beta\left(\Pi_{h} \mathbf{q}-\mathbf{q}\right), \mathbf{G}_{1}^{h}\right)
$$

and

$$
\left(\mathbf{q}^{h}-\Pi_{h} \mathbf{q}, \boldsymbol{\delta}_{2}^{h}\right)=\left(\beta\left(\mathbf{q}-\Pi_{h} \mathbf{q}\right), \mathbf{G}_{2}^{h}\right) .
$$

Thus, it follows from (A.10) and (A.11) that the following relations are valid:

$$
\begin{aligned}
& \left\|u^{h}-P_{h}^{0} u\right\|_{0, \infty} \leq\left|\left(\beta\left(\Pi_{h} \mathbf{q}-\underset{\sim}{q}\right), \mathbf{G}_{1}^{h}\right)\right|, \\
& \left\|\mathbf{q}^{h}-\Pi_{h} \mathbf{q}\right\|_{0, \infty} \leq\left|\left(\beta\left(\mathbf{q}-\Pi_{h} \mathbf{q}\right), \mathbf{G}_{2}^{h}\right)\right|
\end{aligned}
$$




\section{ACKNOWLEDGMENT}

This work contains a part of the author's doctoral thesis. The author would like to thank his advisor, Professor Jim Douglas, Jr., for his guidance and many helpful suggestions pertaining to this work. The author also thanks an anonymous referee and the managing editor for their valuable help and suggestions. The research was supported in part by the U.S. Army Research Office through the Mathematical Sciences Institute of Cornell University.

\section{BIBLIOGRAPHY}

1. I. Babuška, The finite element method with Lagrangian multipliers, Numer. Math. 20 (1973), 179-192.

2. H. Blum, Q. Lin, and R. Rannacher, Asymptotic error expansion and Richardson extrapolation for linear finite elements, Numer. Math. 49 (1986), 11-37.

3. J. H. Bramble and J. Xu, A local post-processing technique for improving the accuracy in mixed finite element approximations, Cornell University, Math. Sci. Inst., Technical Report 88-1.

4. F. Brezzi, On the existence, uniqueness, and approximation of saddle point problems arising from Lagrangian multipliers, RAIRO Anal. Numér. 2 (1974), 129-151.

5. F. Brezzi, J. Douglas, Jr., M. Fortin, and L. D. Marini, Efficient rectangular mixed finite elements in two and three space variables, RAIRO Anal. Numér. 21 (1987), 581-604.

6. F. Brezzi, J. Douglas, Jr., and L. D. Marini, Two families of mixed finite elements for second order elliptic problems, Numer. Math. 47 (1985), 217-235.

7. J. Douglas, Jr., T. Dupont, and M. F. Wheeler, An $L^{\infty}$ estimate and a superconvergence result for a Galerkin method for elliptic equations based on tensor products of piecewise polynomials, RAIRO Anal. Numér. 8 (1974), 61-66.

8. J. Douglas, Jr. and J. E. Roberts, Global estimates for mixed methods for second order elliptic equations, Math. Comp. 44 (1985), 39-52.

9. J. Douglas, Jr. and J. Wang, Superconvergence for mixed finite element methods on rectangular domains, Calcolo 26 (1989), 121-134.

10. R. Durán, Error analysis in $L^{p}, 1 \leq p \leq \infty$, for mixed finite element methods for linear and quasi-linear elliptic problems, RAIRO Anal. Numér. 22 (1988), 371-387.

11. R.E. Ewing, R. Lazarov, and J. Wang, Superconvergence of the velocity along the Gauss lines in mixed finite element methods, in SIAM J. Numer. Anal. (to appear).

12. R.S. Falk and J.E. Osborn, Error estimates for mixed methods, RAIRO Anal. Numér. 14 (1980), 249-277.

13. M. Fortin, An analysis of the convergence of mixed finite element methods, RAIRO Anal. Numér. 11 (1977), 341-354.

14. P. Grisvard, Elliptic problems in nonsmooth domains, Pitman Advanced Publishing Program, London, 1985.

15. Q. Lin and T. Lu, Asymptotic expansion for finite element approximation of elliptic problems on polygonal domains, Proc. Sixth Internat. Conf. Comput. Math. Appl. Sci. Eng., Versailles, 1983.

16. Q. Lin, T. Lu, and S. Shen, Maximum norm estimate, extrapolation and optimal point of stresses for the finite element methods on the strongly regular triangulation, J. Comput. Math. 1 (1983), 376-383.

17. Q. Lin and J. Wang, Some expansions of the finite element approximation, Research Report IMS 15, Chengdu Branch of Academia Sinica, 1984.

18. Q. Lin and J. Xu, Linear finite elements with high accuracy, J. Comput. Math. 3 (1985), 115-133. 
19. Q. Lin and Q. Zhu, Asymptotic expansion for the derivative of finite elements, J. Comput. Math. 2 (1984), 361-363.

20. G. I. Marchuk, Methods of numerical mathematics, 2nd ed., Springer-Verlag, New York, Heidelberg, Berlin, 1982.

21. M. Nakata, A. Weiser, and M. F. Wheeler, Some superconvergence results for mixed finite element methods for elliptic problems on rectangular domains, The Mathematics of Finite Elements and Applications V (J. R. Whiteman, ed.), Academic Press, London, 1985, pp. 367-389.

22. P. A. Raviart and J. M. Thomas, A mixed finite element method for 2 nd order elliptic problems, Mathematical Aspects of Finite Element Methods (I. Galligani and E. Magenes, eds.), Lecture Notes in Math., vol. 606, Springer-Verlag, Berlin and New York, 1977, pp. 292-315.

23. J. Wang, Asymptotic expansions and $L^{\infty}$-error estimates for mixed finite element methods for second order elliptic problems, Numer. Math. 55 (1989), 401-430.

Department of Mathematics, The University of Wyoming, Laramie, Wyoming 82071

E-mail address: junping@outlaw.uwyo.edu 